\title{
OUTPUT AND PRICE LEVEL EFFECTS OF MONETARY UNCERTAINTY IN A MATCHING MODEL
}

\author{
BRETT KATZMAN, JOHN KENNAN, AND NEIL WALLACE
}

\begin{abstract}
Monetary uncertainty and information lags are put into a random matching model so that the resulting setting has some meetings in which producers are relatively informed and others in which consumers are relatively informed.

For that setting, the ex ante socially optimal way to conduct trade is characterized. The optimum can display a variety of relationships between money and total output and the price level. While the price level is always sticky, even the direction of its response and that of total output depend on the magnitude of the lag and on subtle features of the serial correlation properties of the money supply.
\end{abstract}

JEL classification \#'s: E30, E40, D82.

\section{INTRODUCTION}

We embed aggregate monetary shocks and lags in seeing monetary realizations into the random-matching model environment of Shi [1995] and Trejos and Wright [1995]. As regards lags, we assume that some people see monetary realizations as they occur and others see them only with a one-date lag. We use the resulting model to study the behavior of total output and the price level in an ex ante optimum, an optimum that is subject to the assumed monetary shocks and the assumed information structure. While the monetary shocks follow a first-order Markov process, the lag makes the price level and total output follow a second-order process. We characterize analytically the optimum in terms of what happens in the different kinds of meetings - meetings in which both the consumer and the producer are informed about current realizations, those in which neither is informed, and those in which one is informed and the other uninformed. Because the price level and total output are complicated weighted sums of what happens in the different kinds of meetings, we are not able to characterize those aggregates analytically. Therefore, we illustrate some of the possibilities for aggregates by way of several examples.

Date: February, 2001.

We thank Edward J. Green, Nobuhiro Kiyotaki, Narayana Kocherlakota, Rody Manuelli, Nancy Stokey, and many seminar participants for helpful comments. 
The computational scheme we use for examples relies heavily on our analytical characterization of what happens in meetings.

As might be expected, both the main analytical challenge in studying the model and the interesting possibilities for aggregates implied by it are due to the meetings in which one person has superior information about the future value of money. Is it plausible that such differential information is important in actual economies, past and present? Changes in the value of money are a pervasive feature of economies that use money of any kind. If the value of money is constantly changing, then it is hard to believe that everyone has the same information at all times. ${ }^{1}$ In commodity monetary systems, differential information could have arisen from the slow spread of information about discoveries of the commodity - discoveries of gold and silver. More generally, it could arise from the existence of costs of acquiring information. ${ }^{2}$ Differential information could also help explain why so much attention is focused on price stability as a goal of policy. Given differential information, price level instability would naturally inhibit trade, because trade would be viewed as benefiting the better informed at the expense of the less well-informed. In our model, the differential information is stark and is imposed in a way that abstracts from distributional issues: at each date, some randomly selected people are informed about the current realization of the money supply and the rest are informed with a one-date lag. Our focus is primarily on the positive implications of the implied differential information. We study those implications by varying the fraction who are informed without a lag - the benchmark being the case of everyone informed (no differential information).

Our model has several attractive features. First, we use an environment in which the use of money is essential, an environment which was not formulated originally to study the effects of monetary shocks. Nevertheless, the features that make money essential and the differential information fit well together. In order for money to be essential, it is necessary that individual histories are not common knowledge (see Kocherlakota [1998] and Wallace [1998]). Therefore, exogenous changes in individual money holdings can plausibly be part of what is not common knowledge. Also, because trade occurs in informationally separated meetings, there are no commonly observed prices from which people can draw fully revealing inferences about aggregate monetary realizations even if those are the only shocks. Second, we study an ex ante optimum. Most previous work on matching models has assumed a bargaining rule that gives each

\footnotetext{
${ }^{1}$ For example, when an agent who trades frequently exchanges money for goods with an agent who seldom trades, the frequent trader usually has superior information about the value of money in recent transactions.

${ }^{2}$ Jones and Manuelli (2000) show that if it is costly to acquire information, then informational differences may arise even if everyone has access to the same information.
} 
side some fixed share of the "bargaining power." With differential information, there are any number of ways that trade could be conducted in meetings. Rather than adopt an arbitrary one, we analyze the best one in a well-defined class; that is, the society chooses the optimal way to conduct trade in meetings subject to (sequential) individual rationality and truth-telling constraints. Our approach ensures that the relationships between money and other aggregates that we find are not due to having imposed an inefficient way to conduct trade. Third, we separate consumer-producer status from informed-uninformed status. The effects of monetary shocks with information lags were first analyzed rigorously by Lucas (1972). He assumed that producers, the young in his overlapping generations structure, were relatively uninformed. Except in Jones and Manuelli (2000), all subsequent work pursuing the information-lag approach has followed Lucas in assuming that producers are relatively uninformed. ${ }^{3} \mathrm{We}$ treat consumers and producers symmetrically with respect to information. Barro (1989) suggested that such symmetry would overturn the expansionary effects of increases in the quantity of money. ${ }^{4}$ While that is a possibility in our model, our examples suggest that it happens only for increases which are very unlikely.

Our model has one main limitation. While goods are divisible, money is indivisible and each person's holding is limited to be zero or one unit. This restriction on money holdings simplifies the analysis in two ways. Because we do not permit people to commit to randomization in a meeting, the consumer in a meeting either surrenders a unit of money or not, and if not, then there is no trade in that meeting. This simplifies the consequences of truth-telling in the following way. In meetings in which one person is informed and the other uninformed, either there is a pooling outcome in which the trade does not depend on the current state or there is separation, but only of the following sort: there is a single output quantity that is traded for money in some current states and there is no trade in the remaining states. Even more important, the zero-one restriction on money holdings implies that the distribution of money holdings at the start of each date is given by the last realization of the

\footnotetext{
${ }^{3}$ Our model most closely resembles Wallace (1997) and Jones and Manuelli (2000) Wallace because it uses the same background environment and Jones and Manuelli because they permit either consumers or producers to be relatively informed. However, both have a distinct partial equilibrium flavor because both study the effects of one-time uncertainty. Also, both assume given bargaining rules.

4 "Second, the predicted positive effect of surprise money on output and employment was sensitive to changes in specification. Although the belief that a current price or wage is temporarily high represents a perceived profit opportunity for suppliers of goods and labor, it represents a correspondingly bad deal for demanders. Hence a benchmark, symmetric model implies that surprises in money and prices- even if they are substantial because of imperfect information- leave output and employment unchanged (see Barro and King, 1984)" [page 2].
} 
assumed Markov process for the money supply and, therefore, is exogenous. With richer individual money holdings, there would be interaction between the trades and the distribution. As a crude way to control for the effects of restricting individual money holdings to be zero or one unit, we discuss results relative to the benchmark in which everyone is informed about the current realization when it occurs.

Relative to the benchmark, the model implies two sources of price-level stickiness. (Because one unit of money exchanges for some output in a meeting, the price in a meeting is the inverse of output in the meeting. The price level is defined to be the implied total output deflator.) First, when two uninformed people meet, the price in that meeting cannot depend on the current realization. Second, when one trader is relatively informed, that information cannot affect the price in the meeting without violating the truth-telling constraints. As for total output, relative to the benchmark it responds positively to an increase in the amount of money except when that increase is sufficiently unlikely. Then negative impact effects can occur.

Although our information structure treats producers and consumers symmetrically, the impact effects of increases and decreases in the amount of money are not symmetric, even when the Markov process for the money supply is symmetric. If a high monetary realization is sufficiently unlikely, then the optimum has no-trade in meetings between informed producers and uninformed consumers when such a realization occurs (because it is then possible to have higher output when the unlikely realization does not occur). On the other hand, for the range of parameter values that we consider, the optimum does not have no-trade when a monetary decrease occurs. Empirical studies of the money-output relationship typically use vector autoregressions that impose symmetrical effects of increases and decreases in money. In contrast, the appropriate econometric specification for our model is a Markov chain.

The paper is organized as follows. In section 2, we describe the environment. In section 3, we present a preview of the analysis and results. In section 4 , we describe the mechanisms we consider and the optimum problem. In section 5, we present a characterization of the optimum. Section 6 contains the examples. Section 7 contains a discussion of randomization, while section 8 contains a discussion of the relationship between the optimum and the outcome for bargaining in which consumers make take-it-or-leave-it offers.Proofs are given in the Appendix.

\section{The ENVIRONMENT}

Aside from the uncertainty about the stock of money, the environment is that in Wallace (1997), which, in turn, is essentially that in Shi (1995) and Trejos and Wright (1995). Time is discrete and the horizon is infinite. There 
are $N$ distinct, divisible, and perishable types of goods at each date and there is a $[0,1]$ continuum of each of $N$ specialization types of people, where $N \geq$ 3. A specialization type- $n$ person consumes only good $n$ and produces only good $n+1(\operatorname{modulo} N)$, for $n=1,2, \ldots, N$. Each person maximizes expected discounted utility with discount factor $\beta \in(0,1)$. Utility in a period is given by $u(x)-y$, where $x$ is the amount consumed and $y$ is the amount produced. The function $u$ is defined on $[0, \infty)$, is increasing, strictly concave, and satisfies $u(0)=0, u^{\prime}(0)=\infty$, and $u^{\prime}(\infty)<1$. In each period, people are randomly matched in pairs. Meetings are of two sorts: single-coincidence meetings, those between a type $n$ person (the producer) and a type $n+1$ person (the consumer) for some $n$; and no-coincidence meetings, those in which neither person produces what the other consumes. (Because the number of types, $N$, exceeds two, there are no double-coincidence meetings.) We assume that people cannot commit to what they will do in future meetings. Money consists of perfectly durable and indivisible objects which cannot be produced and which do not yield utility directly. We assume that each person can carry from one date to the next at most one unit of money. We also assume that each trader in a meeting is able to see the trading partner's specialization type, money holdings, and whether the person is informed or not, but is otherwise ignorant about the trading partner's history.

The quantity of money follows an $S$-state Markov process. That is, there are $S$ potential levels for the stock of money: $m_{1}, m_{2}, \ldots, m_{S}$ where $m_{i} \in\left(0, \frac{1}{2}\right]$ and $m_{i}<m_{i+1}$. Here $m_{i}$ is the state $i$ amount of money per specialization type. ${ }^{5}$ We let $\pi_{i j}$ denote the probability that the current state is $j$ given that the previous state is $i$ and let $\Pi$ denote the associated transition matrix. We assume that $\pi_{i j}>0$ which implies that $\Pi$ has a unique invariant distribution which assigns positive probability to each state.

We want changes in the money supply to come about in a way that gives no immediate information to the uninformed. That is accomplished by having only informed people experience transfers of money in the following way. ${ }^{6}$ At the end of each date after meetings have dissolved, the current amount of money is publicly announced. (Consequently, at that time, people differ only in money holdings, not in information.) Then, a randomly chosen subset of each specialization type, of measure $\lambda$, is selected and is informed about the new state. If the previous state is $i$, then the measure of newly informed with

\footnotetext{
${ }^{5}$ The upper bound on $m_{i}$ assures that increases in the amount of money do not, by themselves, crowd out trade. That possibility arises only because of the bound on individual holdings. Therefore, it seems best to preclude it by limiting the support as we do.

${ }^{6}$ If everyone could experience transfers, then we would have to distinguish additional types; in particular, we would have to distinguish between uninformed who experience a transfer and uninformed who do not. Although that specification would fit better with exogenous changes in the quantity of money, the added complexity did not seem worthwhile.
} 
money is $m_{i} \lambda$ and the measure without money is $\left(1-m_{i}\right) \lambda$. If the new state is $j>i$, then a randomly chosen subset of the informed without money, a subset of measure $m_{j}-m_{i}$, is given a unit of money. If the new state is $j<i$, then a randomly chosen subset of the informed with money, a subset of measure $m_{i}-m_{j}$, loses a unit of money. Then meetings occur and the sequence is repeated.

The following table shows the fraction of each specialization type according to whether they are informed and whether they have money given the previous and current states.

Table 1. Distribution when the previous state is $i$ and the current state is $j$.

\begin{tabular}{|c|c|c|c|}
\hline & 0 units of money & 1 unit of money & sums \\
\hline informed & $\lambda\left(1-m_{i}\right)-\left(m_{j}-m_{i}\right) \equiv \theta_{j i}$ & $\lambda m_{i}+m_{j}-m_{i} \equiv \lambda-\theta_{j i}$ & $\lambda$ \\
\hline uninformed & $(1-\lambda)\left(1-m_{i}\right)$ & $(1-\lambda) m_{i}$ & $1-\lambda$ \\
\hline sums & $1-m_{j}$ & $m_{j}$ & 1 \\
\hline
\end{tabular}

In order to be able to have all changes in the amount of money be experienced by those who are informed, we need to assume that $\lambda$ is large enough relative to the monetary changes. The relevant bound is $\lambda \geq \frac{m_{S}-m_{1}}{m_{S}} .^{7}$

The monetary uncertainty has obvious incentive effects in our model. It makes those without money, the potential producers, less willing to produce in order acquire money because (i) they may lose the money acquired before they get to spend it; and (ii) if they do not produce, then they may be given a unit. It also makes those with money, the potential consumers, more willing to spend money because (i) if they do not spend it, then they may lose it; and (ii) if they do spend it, then they may be given a unit.

\section{Preview of the ANALysis And Results}

We study a class of deterministic mechanisms which can be described as follows. At each meeting, there is a computer that is programmed at date 0 before people go off to their meetings, but is able to receive messages. At the beginning of a date, when the previous state is publicly announced, that information is received by each computer. In addition, the computer at a meeting sees who is informed and who is a producer and who is a consumer. If both are informed, then both simultaneously announce to the computer a possible current state. If one person is informed, then that person announces to the computer a possible state. The uninformed person in the meeting does not see the announcement. In all cases, the computer then proposes a trade,

\footnotetext{
${ }^{7}$ This lower bound on $\lambda$, together with the assumption that $m_{S} \leq \frac{1}{2}$ implies $\left(1-m_{i}\right) \lambda$ $\geq\left(1-m_{S}\right) \lambda \geq m_{S} \lambda \geq m_{S}-m_{1} \geq m_{j}-m_{i}$. Thus $\theta_{j i} \geq 0$. Also, $(1-\lambda) m_{i} \leq(1-\lambda) m_{S}=$ $m_{S}-\lambda m_{S} \leq m_{S}-\left(m_{S}-m_{1}\right) \leq m_{j}$, where the second inequality follows from the lower bound on $\lambda$. This implies that $m_{j} \geq(1-\lambda) m_{i}$, and, hence, that $\lambda-\theta_{j i}$ is non-negative.
} 
which may be no trade. Then each person's choice is either to accept or reject, where rejection by either person implies no trade. Acceptance by both implies that the proposed trade is carried out. Whether trade occurs or not, the meeting ends. A mechanism is incentive feasible if it induces truth-telling and if the proposed trade satisfies individual rationality, and, therefore, induces acceptance. $^{8}$

Because the mechanisms are deterministic, output in a meeting is positive if and only if money is transferred. It follows that the only meetings in which trade can occur are single-coincidence meetings in which the consumer has money and the producer does not. We call these trade meetings. We begin by pointing out the distinct potential roles of the current money supply. The amount of money has a direct effect on the number of trade meetings; in particular, because the amount of money is no greater than $\frac{1}{2}$, the number of trade meetings, which is proportional to $\left(1-m_{s}\right) m_{s}$, is increasing in $m_{s}$. This is the extensive-margin effect of the current money supply. In contrast, the current money supply has only an indirect effect on what happens in trade meetings, the intensive margins. Because, by assumption, there is one unit of money in each trade meeting, any effect on what happens in trade meetings is due to the effect of the current money supply on expectations: potential producers think about how valuable any acquired money will be in the future, while potential consumers compare the current state and future states as alternative times to spend money. It follows that the serial correlation properties of $\Pi$ are critical; in particular, there would be no intensive margin effects if the money supply process was i.i.d. However, serial correlation is not sufficient for intensive-margin effects. To see why, it is helpful to examine the optimum problem for the case of alternative constant money supplies.

Suppose the money supply $m_{s}$ is known and constant. Then our mechanisms reduce to ones with a constant amount $y$ produced in every trade meeting. With no differential information, the only constraints are individual rationality (IR) constraints, one for the producer and one for the consumer. Let $V_{k}$ be the discounted expected utility at the start of a period associated with holding $k$ units of money, where $k=0,1$. Then $V_{0}=\beta V_{0}+\frac{m_{s}}{N}\left(-y+\beta\left[V_{1}-V_{0}\right]\right)$ and $V_{1}=$ $\beta V_{1}+\frac{1-m_{s}}{N}\left(u(y)-\beta\left[V_{1}-V_{0}\right]\right)$. These two equations are linear in the $V_{k}$ and can be solved uniquely for them in terms of $y$. Let $\Delta(y)$ denote the implied solution for $V_{1}-V_{0}$. The producer's $I R$ constraint is $y \leq \beta \Delta(y)$, while the consumer's

\footnotetext{
${ }^{8}$ Note that the ex post stage here is taken to be the situation at the end of a meeting, rather than the subsequent stage at which the current money supply is publicly announced (which is the point at which ex post welfare comparisons would conventionally be made). If it is assumed that all information available to the participants in each meeting is revealed in that meeting, then there are additional restrictions on the set of mechanisms. We are indebted to Nobuhiro Kiyotaki and Narayana Kocherlakota for conversations that helped steer us away from such a more restrictive specification.
} 


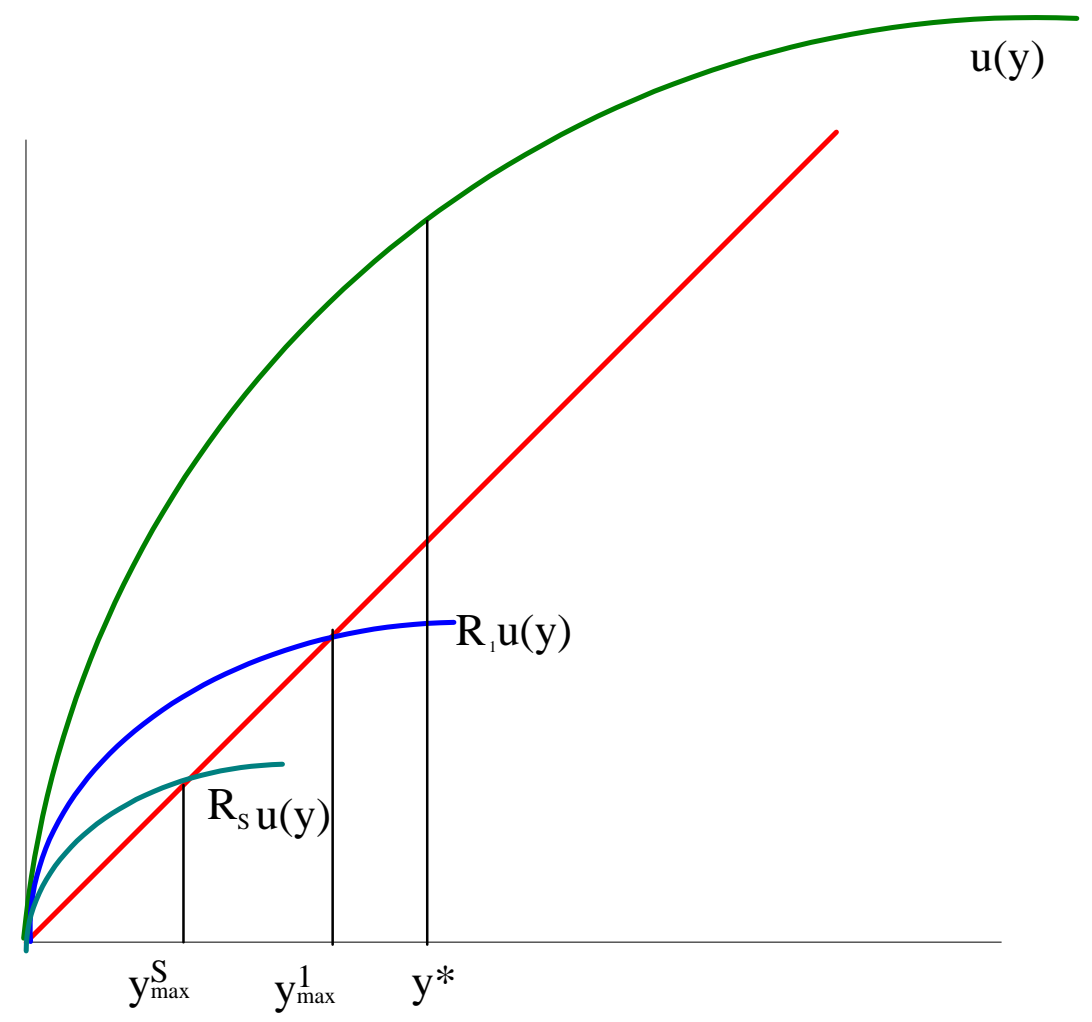

FiguRe 1

is $\beta \Delta(y) \leq u(y)$. Two facts are easily derived. First, satisfaction of the the producer's IR constraint implies satisfaction of the consumer's. (After all, the producer experiences current disutility for a probability of consuming the same amount in the future. Hence, $u(y)$ must exceed $y$ by a sufficient margin. The consumer, though, is in the opposite situation, so $u(y) \geq y$ is sufficient for the consumer's IR constraint.) Second, the producer's IR constraint is satisfied for all $y$ that satisfy $y \leq y_{\max }^{s}$, where $y_{\max }^{s}$ is the unique positive solution to $y=\beta \Delta(y)$; namely, the unique positive solution to

$$
R_{s} u(y)=y \text {. }
$$

where $R_{s}$, the discount factor for a claim that will be redeemed at the next consumption opportunity, is given by $\frac{1}{R_{s}}=1+\frac{(1-\beta) N}{\beta\left(1-m_{s}\right)}$.

This situation is illustrated in Figure 1. Evidently, $y_{\max }^{s}$ is decreasing in $m_{s}$, $N$, and $\frac{1}{\beta}$, and $y_{\max }^{s}$ approaches 0 as either $N$ or $\frac{1}{\beta}$ goes to infinity. Ex ante utility, our objective, is $\left(1-m_{s}\right) V_{0}+m_{s} V_{1}$, which is equal to $\frac{m_{s}\left(1-m_{s}\right)}{(1-\beta) N} z(y)$, where $z(y) \equiv u(y)-y$. Therefore, the optimum problem in this case is to choose $y$ to maximize $z(y)$ subject to $y \leq y_{\max }^{s}$. Let $y^{*}$ denote the unconstrained maximum 
of $z$; namely, the unique solution to $u^{\prime}\left(y^{*}\right)=1$. Then the solution to the optimum problem is $\min \left(y_{\max }^{s}, y^{*}\right)$. Notice that if $y_{\max }^{s}>y^{*}$, then the solution is $y^{*}$ and does not depend on $m_{s}$. If, instead, $y_{\max }^{s}<y^{*}$, then the solution is $y_{\max }^{s}$. To avoid the uninteresting solution in which output in every trade meeting is $y^{*}$ in the complete model, we assume throughout that $y_{\max }^{1} \leq y^{*}$ (a condition that can always be met by making $\beta$ small or by making $N$ large). ${ }^{9}$ As we show below, this is sufficient to ensure that output in any meeting is less than $y^{*}$.

As we also show below, that upper bound on output implies that the objective for the complete model is increasing in outputs. Therefore, the bound tends to produce an optimum with binding producer IR constraints. For the case of a constant money supply, as noted above, it follows that the consumer IR constraint is slack. For the complete model, we impose parameter restrictions so that binding producer IR constraints imply slack consumer IR constraints. That is achieved, as we show, by assuming that the range of the support for the money supply is not too large. ${ }^{10}$

It is under the above two assumptions $\left(y_{\max }^{1} \leq y^{*}\right.$ and slack consumer IR constraints) that we characterize the optimum. The conjectures about the optimum in the general case come from thinking about the partial equilibrium problem in which trades do not affect the future value of money. Because exante welfare is increasing in output in every kind of meeting, if consumer IR constraints never bind and if there were no truth-telling constraints, then an optimum would satisfy producer IR constraints with equality (that is, all of the gains from trade would accrue to the consumer). However, there are truthtelling constraints for meetings in which one person is relatively informed. Because the amount of money that changes hands is always one unit, the amount produced in exchange for money in such meetings cannot depend on the current state. (If it did, then it would not be consistent with truth-telling by the informed person.) Therefore, for such meetings, the mechanism has only to describe the amount produced and a partition of the set of current states into trade and no-trade sets. Moreover, those sets have to be ordered: if the producer is informed, then the set of states in which trade occurs has to be

\footnotetext{
${ }^{9}$ The possibility that output is constant at $y^{*}$ whenever trade occurs is an artifact of the unit upper bound on holdings. If money is divisible or even if individuals can hold many units of indivisible money and if expected discounted utility is strictly increasing in money holdings, then output equal to $y^{*}$ would fail to satisfy producer $i r$ constraints in meetings between producers with sufficiently high money holdings and consumers with sufficiently low money holdings.

${ }^{10}$ This assumption rules out an interesting possibility; namely, that a sufficiently large and rare decrease in the amount of money produces a large decline in output because it is optimal for there to be no trade in such states when informed consumers meet uninformed producers. Our assumption rules out large changes.
} 
composed of those in which acquiring a unit of money is most valuable; if the consumer is informed, then it has to be composed of those in which acquiring a unit of money is least valuable.

If the consumer is relatively informed and there is trade in all states, then the largest possible output is that consistent with the producer's participation constraint, given the information available to the producer (which includes the previous state and the observation that the consumer has money). Could it, instead, be worthwhile to have zero output in some states so as to have higher output in other states? Because the consumer is informed, truth-telling implies that the value of money would have to be higher when output is zero. But excluding such high-value states reduces the expected value of the money received by the producer, and, therefore, leads to lower output. Thus, it is undesirable to exclude states in this way.

If the producer is relatively informed and there is trade in all states, then the largest possible output is that consistent with the producer's participation constraint for the minimal value of acquiring money. If, instead, output is zero in states in which the value of money is low, then it is possible to increase output in the other states without violating the producer's participation constraint. Hence, it is desirable to set output to zero in the low-value states if they are unlikely enough given the previous state.

The above considerations led us to the following conjecture about the optimum for the complete model. It is optimal to have trade occur in every trade meeting except possibly in some of the meetings between informed producers and uninformed consumers. Moreover, in all meetings, there are binding producer individual-rationality constraints. We substantiate this claim in the next two sections.

\section{Mechanisms And The optimum PROBlem}

The mechanism design problem is presented in three steps. First, we describe a set of deterministic Markov mechanisms. Second, we describe the subset of mechanisms that we call incentive-feasible. Lastly, we describe our selection from the set of incentive-feasible mechanisms.

In general, the outcome of a trade meeting depends on how the consumer and the producer bargain over the quantity of output that is exchanged for a unit of money. For any given bargaining procedure, the outcome is an equilibrium of a game in which the players' strategies may depend on the history of previous states. In our environment, it is natural to restrict attention to strategies that depend on history only to the extent that it helps to predict the amount of money in future periods: that is, strategies that depend only on the current state and the previous state. Then the Revelation Principle implies

that there is no further loss of generality if we consider only direct revelation 
mechanisms: those in which the producer and consumer in a trade meeting are asked to say what they know about the current state, and in which the outcome depends on these statements and on the previous state. For technical reasons, we impose the further restriction that the outcome of each meeting is a deterministic function of the announcements and the previous state; this restriction is discussed further in Section 7 below.

Let $\mathbb{S}_{+} \equiv\{1,2, \ldots, S\}$ and $\mathbb{S} \equiv \mathbb{S}_{+} \cup\{0\}$. We use $\mathbb{S}$ to denote the set of individual information states, where $s=0$ means uninformed about the current state and $s>0$ means informed and that the current amount of money is $m_{s}$. We define a (Markov) mechanism as follows:

Definition 1. A mechanism is a pair $(y, a)$ where $y: \mathbb{S} \times \mathbb{S} \times \mathbb{S}_{+} \rightarrow R_{+}$and $a: \mathbb{S} \times \mathbb{S} \times \mathbb{S}_{+} \rightarrow\{0,1\}$.

Here $y\left(s^{c}, s^{p}, i\right)$ denotes output in a trade meeting when the consumer announces information state $s^{c}$, the producer announces $s^{p}$, and the previous money-supply realization is $i$, and $a\left(s^{c}, s^{p}, i\right)$ denotes the money transfer $(0$ means no transfer, 1 means transfer) in such a meeting.

In order to express the constraints on mechanisms implied by our specification of incentive-feasibility, it is helpful to have a notation for expected discounted utilities. We let $V_{k}(i)$ denote the expected utility of someone who has $k$ units money just after the previous state $i$ is announced (and before the determination of the new state and the new set of informed people). We let $V_{k}$ denote the $S$-element vector with generic component $V_{k}(i)$. We also let $\Delta_{i} \equiv V_{1}(i)-V_{0}(i)$ and $\Delta \equiv V_{1}-V_{0}$. We show in the Appendix that each mechanism $(y, a)$ implies unique expected utilities $\left\{V_{0}, V_{1}\right\}$ and, hence, a unique $\Delta$. Therefore, when convenient, we write $\Delta(y, a)$ to express the dependence of $\Delta$ on $(y, a)$.

It is also helpful to let $G_{k}\left(\iota^{c}, \iota^{p}, s, i\right)$ be the gain, relative to not trading, in a trade meeting of someone with $k$ units of money when the current realization is $s$ and the previous realization is $i$, and when both parties announce truthfully. Here, $\iota^{c} \in\{0,1\}$ indicates whether the consumer is informed $\left(\iota^{c}=1\right)$ or uninformed $\left(\iota^{c}=0\right)$, and $\iota^{p} \in\{0,1\}$ indicates, in the same way, whether the producer is informed. Then,

$$
\begin{aligned}
& G_{0}\left(\iota^{c}, \iota^{p}, s, i\right)=-y\left(\iota^{c} s, \iota^{p} s, i\right)+a\left(\iota^{c} s, \iota^{p} s, i\right) \beta \Delta_{s} \\
& G_{1}\left(\iota^{c}, \iota^{p}, s, i\right)=u\left(y\left(\iota^{c} s, \iota^{p} s, i\right)\right)-a\left(\iota^{c} s, \iota^{p} s, i\right) \beta \Delta_{s} .
\end{aligned}
$$

Notice that

$$
G_{0}\left(\iota^{c}, \iota^{p}, s, i\right)+G_{1}\left(\iota^{c}, \iota^{p}, s, i\right)=z\left(y\left(\iota^{c} s, \iota^{p} s, i\right)\right)
$$


where $z(y) \equiv u(y)-y .^{11}$

We can use these gain definitions to express the truth-telling and IR constraints. For trade meetings with symmetric information, the IR constraints are

$$
\begin{aligned}
& G_{0}(1,1, s, i) \geq 0 \text { and } \sum_{j=1}^{S} \pi_{i j} G_{0}(0,0, j, i) \geq 0 \\
& G_{1}(1,1, s, i) \geq 0 \text { and } \sum_{j=1}^{S} \pi_{i j} G_{1}(0,0, j, i) \geq 0
\end{aligned}
$$

where (4.3) refers to producers, and (4.4) refers to consumers. In each case, the first inequality pertains to an informed person who meets an informed person, while the second pertains to an uninformed person who meets an uninformed person. (Of course, for uninformed people, the condition is that there be expected gains from trade because the trade occurs before the participants know the current state.) When both are informed, truth-telling is always a best response (provided the mechanism proposes no-trade when it receives mutually inconsistent reports). Hence, truth-telling in meetings without differential information does not imply additional constraints.

We next consider truth-telling constraints in trade meetings between the informed and the uninformed. For all $\left(s, s^{\prime}\right) \in \mathbb{S}_{+} \times \mathbb{S}_{+}$, we require

$$
\begin{gathered}
a(0, s, i) \beta \Delta_{s}-y(0, s, i) \geq a\left(0, s^{\prime}, i\right) \beta \Delta_{s}-y\left(0, s^{\prime}, i\right) \\
u(y(s, 0, i))-a(s, 0, i) \beta \Delta_{s} \geq u\left(y\left(s^{\prime}, 0, i\right)\right)-a\left(s^{\prime}, 0, i\right) \beta \Delta_{s}
\end{gathered}
$$

where (4.5) refers to informed producers, and (4.6) refers to informed consumers. Thus, as noted above, if $a(0, s, i)=a\left(0, s^{\prime}, i\right)$, then the producer's constraint implies $y(0, s, i)=y\left(0, s^{\prime}, i\right)$; while if $a(s, 0, i)=a\left(s^{\prime}, 0, i\right)$, then the consumer's constraint implies $y(s, 0, i)=y\left(s^{\prime}, 0, i\right)$. Therefore, we let

$$
S^{p}(i)=\left\{j \in \mathbb{S}_{+}: a(0, j, i)=1\right\} \text { and } S^{c}(i)=\left\{j \in \mathbb{S}_{+}: a(j, 0, i)=1\right\}
$$

and let $y(0, j, i)=Y^{p}(i)$ for $j \in S^{p}(i)$ and $y(j, 0, i)=Y^{c}(i)$ for $j \in S^{c}(i)$. Then the truth-telling constraints are equivalent to

$$
\begin{gathered}
\max _{j \in \mathbb{S}_{+}-S^{p}(i)}\left\{\beta \Delta_{j}\right\} \leq Y^{p}(i) \leq \min _{j \in S^{p}(i)}\left\{\beta \Delta_{j}\right\} \\
\max _{j \in S^{c}(i)}\left\{\beta \Delta_{j}\right\} \leq u\left(Y^{c}(i)\right) \leq \min _{j \in \mathbb{S}_{+}-S^{c}(i)}\left\{\beta \Delta_{j}\right\}
\end{gathered}
$$

where we interpret (4.8) to be vacuous if $S^{p}(i)$ or $\mathbb{S}_{+}-S^{p}(i)$ is empty and (4.9) to be vacuous if $S^{c}(i)$ or $\mathbb{S}_{+}-S^{c}(i)$ is empty. These are the truth-telling constraints with regard to the partition of current states between the no-trade

\footnotetext{
${ }^{11}$ The expression for $G_{0}$ follows from $G_{0}=-y+a \beta V_{1}+(1-a) \beta V_{0}-\beta V_{0}$ and similarly for $G_{1}$.
} 
states and the trade states. They describe the sense in which the gain from acquiring money has to be ordered across trade and no-trade states.

Finally, we have IR constraints for participants in these meetings. For an informed producer, the IR constraint is the second inequality in (4.8), while for an uninformed producer, it is

$$
Y^{c}(i) \leq \frac{\sum_{j \in S^{c}(i)} \pi_{i j}\left(\lambda-\theta_{j i}\right) \beta \Delta_{j}}{\sum_{k \in S^{c}(i)} \pi_{i k}\left(\lambda-\theta_{k i}\right)}
$$

For an informed consumer, the IR constraint is the first inequality in (4.9), while for an uninformed consumer, it is

$$
\frac{\sum_{j \in S^{p}(i)} \pi_{i j} \theta_{j i} \beta \Delta_{j}}{\sum_{k \in S^{p}(i)} \pi_{i k} \theta_{k i}} \leq u\left(Y^{p}(i)\right)
$$

The probability weights in these expressions represent the beliefs of an uninformed person, given the information implied by truth-telling, which reveals the set of trade states, and the knowledge about the current state implied by observing the money holding of someone who could have gained or lost money.

We can now record the definition of an incentive-feasible mechanism.

Definition 2. A mechanism $(y, a)$ is incentive feasible if there exists $\left(V_{0}, V_{1}\right)$ that satisfies (4.1)-(4.11).

An immediate implication is that if $(y, a)$ is incentive-feasible (in particular, satisfies the IR constraints), then $V_{0}$ and $V_{1}$ are non-negative.

We now describe our selection from the above set of incentive-feasible mechanisms. Our selection maximizes ex ante utility, denoted $Z$, which is defined as

$$
Z=\sum_{s=1}^{S} p_{s}\left[m_{s} V_{1}(s)+\left(1-m_{s}\right) V_{0}(s)\right] .
$$

Here $p_{i}$ denotes the invariant probability, implied by $\Pi$, that the amount of money is $m_{i}$. This objective corresponds to starting up the economy by drawing the previous state from that invariant distribution and considering expected utility prior to such a draw and to initial assignments of money holdings. The following lemma expresses $Z$ in terms of the parameters of the environment and the output levels in an arbitrary mechanism. 
Lemma 1. For any mechanism,

$$
\begin{aligned}
Z(1-\beta) N= & (1-\lambda)^{2} \sum_{i=1}^{S} p_{i} m_{i}\left(1-m_{i}\right) z[y(0,0, i)] \\
& +\sum_{i=1}^{S} p_{i} \sum_{s=1}^{S} \pi_{i s} \theta_{s i}\left(\lambda-\theta_{s i}\right) z[y(s, s, i)] \\
& +(1-\lambda)\left\{\sum_{i=1}^{S} p_{i} m_{i} \sum_{s=1}^{S} \pi_{i s} \theta_{s i} z[y(0, s, i)]\right. \\
& \left.+\sum_{i=1}^{S} p_{i}\left(1-m_{i}\right) \sum_{s=1}^{S} \pi_{i s}\left(\lambda-\theta_{s i}\right) z[y(s, 0, i)]\right\} .
\end{aligned}
$$

Notice that output levels, the components of $y$, appear in $Z$ only by way of the function $z$ and that $a$, the money transfer variable, does not appear. Thus $Z$ is an average of the joint gains from trade in the various kinds of meetings, weighted by the relative frequencies of these meetings.

It is not difficult to show that an optimal mechanism exists. Recall that a mechanism $(y, a)$ is a pair of functions with finite domain: in particular, $y$ can be regarded as a finite dimensional vector. Because $z\left(y^{*}\right)$, where $u^{\prime}\left(y^{*}\right)=$ 1 , is an upper bound on $z\left(\left(y\left(s, s^{\prime}, i\right)\right)\right.$ and because the weights in (4.13) are non-negative, an upper bound on $Z$ is obtained by having $y^{*}$ produced and consumed in all trade meetings. Because one-quarter of all single-coincidence meetings, the maximum of the function $m(1-m)$ for $m \in\left[0, \frac{1}{2}\right]$, is an upper bound on the number of trade meetings, it follows that an upper bound on $Z$ is $\frac{z\left(y^{*}\right)}{4 N(1-\beta)} \equiv Z^{*}$. This implies a bound on the set of incentive-feasible outputs. Let $q=\min _{s}\left(p_{s} m_{s}\right)$. By (4.12), if $(y, a)$ is incentive-feasible, then $V_{1}(s) \leq Z^{*} / q$ and $\Delta_{s} \leq Z^{*} / q$, by the non-negativity of the $V_{k}(s)$. By the IR constraints, it follows that each component of an incentive-feasible $y$ is bounded above by $\beta Z^{*} / q$. Moreover, because the $V_{k}(s)$ and, therefore, $\Delta_{s}$ are continuous in $y$, and because the constraints are expressed as weak inequalities, it follows that the optimum problem amounts to maximizing a continuous function over a non empty compact set. ${ }^{12}$ Therefore, a maximum exists.

\section{A Partial Characterization of the Optimum}

As noted above, we concentrate our study of optima in a region of the parameter space that is both interesting and tractable; namely, that for which

\footnotetext{
${ }^{12}$ Non-emptiness is implied by the fact that no trade satisfies all the constraints. Note that the Theorem of the Maximum does not apply because the constraint set is not lower hemicontinuous.
} 
$y^{*} \geq y_{\max }^{1}$ and $u\left(\beta \min _{s} \Delta_{s}\right) \geq y_{\max }^{1} \cdot{ }^{13}$ The following lemma, which uses the first assumption, shows that satisfaction of producer IR constraints implies that $y_{\max }^{1}$ is an upper bound on $\beta \Delta_{i}(y, a)$ and on output.

Lemma 2. If $(y, a)$ satisfies producer IR constraints, then $y\left(s, s^{\prime}, i\right) \leq \beta \max _{i} \Delta_{i}(y, a) \leq$ $y_{\max }^{1}$.

One consequence is that the objective $Z$ is increasing in each component of an incentive-feasible $y$. Another is that our second assumption, $u\left(\beta \min _{s} \Delta_{s}\right) \geq$ $y_{\max }^{1}$, implies $u\left(\beta \min _{s} \Delta_{s}\right) \geq \beta \max _{s} \Delta_{s}$, which, in turn, implies that consumer IR constraints are not binding at an optimum.

Our main result is Proposition 1, which gives necessary conditions for an optimum.

Proposition 1. If $(y, a)$ is optimal and satisfies $u\left(\beta \min _{s} \Delta_{s}(y, a)\right) \geq y_{\max }^{1}$, then $(i) S^{c}(i) \equiv \mathbb{S}_{+}$,

$$
\begin{gathered}
Y^{c}(i)=\frac{\sum_{j \in \mathbb{S}_{+}} \pi_{i j}\left(\lambda-\theta_{j i}\right) \beta \Delta_{j}(y, a)}{\sum_{j \in \mathbb{S}_{+}} \pi_{i j}\left(\lambda-\theta_{j i}\right)} \text { for all } i \in \mathbb{S}_{+}, \\
a(0,0, i)=1 \text { and } y(0,0, i)=\sum_{j=1}^{S} \pi_{i j} \beta \Delta_{j}(y, a) \text { for all } i \in \mathbb{S}_{+}, \\
a(j, j, i)=1 \text { and } y(j, j, i)=\beta \Delta_{j}(y, a) \text { for all }(i, j) \in \mathbb{S}_{+} \times \mathbb{S}_{+},
\end{gathered}
$$

and

$$
Y^{p}(i)=\min _{j \in S^{p}(i)}\left\{\beta \Delta_{j}(y, a)\right\} \text { for all } i \in \mathbb{S}_{+} ;
$$

(ii) for each $i, S^{p}(i)$ is not empty; and (iii) if $\Delta_{j}(y, a)=\Delta_{k}(y, a)$, then for each $i$ either $j, k \in S^{p}(i)$ or $j, k \in \mathbb{S}_{+}-S^{p}(i)$.

The rather lengthy proof proceeds by contradiction. We first suppose that $(y, a)$ is optimal, but does not satisfy (i). Then we consider $\left(y^{\prime}, a^{\prime}\right)$ given by (i), but with $\Delta(y, a)$ inserted on the right-hand sides of (5.1)-(5.4). Then incentive feasibility of $y$ implies that $y^{\prime} \geq y$. The main part of the proof involves showing that $\Delta\left(y^{\prime}, a^{\prime}\right) \geq \Delta(y, a)$. Given that inequality and lemma 2 , it follows that $\left(y^{\prime}, a^{\prime}\right)$ satisfies all the constraints except possibly truth-telling for producers. If it does, then we have a contradiction because $y_{\max }^{1} \geq y^{\prime} \geq y$ and we have either increased output or replaced no-trade by trade, both of which increase $Z$. If not, then there is some previous state $i$ and some current state $r$ such that the producer would like to produce and acquire money; namely, $\beta \Delta_{r}\left(y^{\prime}, a^{\prime}\right)>Y^{p}(i)^{\prime} \geq \beta \Delta_{r}(y, a)$. We then consider $\left(y^{\prime}, a^{\prime \prime}\right)$, where $a^{\prime \prime}$

\footnotetext{
${ }^{13}$ Later, we present a sufficient condition in terms of parameters - essentially, a limitation on the range of the support for the money supply - for satisfaction of $u\left(\beta \min _{s} \Delta_{s}\right) \geq y_{\max }^{1}$, the assumption that implies that consumer ir constraints are slack.
} 
differs from $a^{\prime}$ only in adding state $r$ to $S^{p}(i)$, the set of trade states. We can show that $\Delta\left(y^{\prime}, a^{\prime \prime}\right) \geq \Delta(y, a)$. Repetition of this argument at most $S$ times leads to trade in every state, which renders truth-telling vacuous. Parts (ii) and (iii) are proved using simple versions of the same argument.

The next lemma shows that for a given specification of the $S^{p}(i)$ sets there is a unique monetary mechanism (i.e., a mechanism with positive trade) that satisfies condition (i) of Proposition 1.

Lemma 3. Given $S^{p}(i)$ for each $i \in \mathbb{S}_{+}$, there exists a unique monetary mechanism that satisfies (5.1)-(5.4).

The proof shows that a monotone and concave mapping whose fixed points coincide with solutions to (5.1)-(5.4) has a unique positive fixed point.

Proposition 1 and lemma 3 give us a simple procedure for finding the optimum for a given environment (that satisfies the hypotheses of Proposition 1). For each specification of the $S^{p}(i)$ sets, obtain the unique monetary mechanism that satisfies (5.1)-(5.4). Discard those that are inconsistent with truth-telling for the informed producer. Among those that remain, the optimum is the one that gives the highest $Z$. Because $S^{p}(i) \equiv \mathbb{S}_{+}$satisfies truth-telling, there is at least one such monetary mechanism which is incentive-feasible.

We now show that the maximized objective is continuous in $\Pi$ provided that $\pi_{i i}$ is bounded away from 0 for each $i$. We establish such continuity in two steps. First, we show that for given $S^{p}(i)$ sets, the unique monetary mechanism that satisfies (5.1)-(5.4) is continuous in $\Pi$.

Lemma 4. Let $S^{p}(i)$ for $i \in \mathbb{S}_{+}$be given. Let $\mathbb{P}=\left\{\Pi: \pi_{i i} \geq \alpha>0\right.$ for all $\left.i\right\}$. Then the unique monetary mechanism that satisfies (5.1)-(5.4) is continuous in $\Pi$ for $\Pi \in \mathbb{P}$.

Now we give the main continuity result.

Proposition 2. If the optimum satisfies $u\left(\beta \min _{s} \Delta_{s}\right) \geq y_{\max }^{1}$, then the maximized objective is continuous in $\Pi$ for $\Pi$ such that $\pi_{i j}$ is positive and $\pi_{i i}$ is bounded away from 0.

While the proof uses lemma 4, it must do more than appeal to the fact that the maximum of continuous functions is continuous. The unique monetary mechanism that satisfies (5.1)-(5.4) for given $S^{p}(i)$ sets may satisfy truthtelling for informed producers for some $\Pi^{\prime} s$ and not others. We can show that if truth-telling holds at $\Pi_{0}$ but not in the neighborhood of $\Pi_{0}$, then condition (iii) in Proposition 1 fails at $\Pi_{0}$. That, in turn, implies that the maximum cannot be at such a point. That and lemma 4 imply that the maximized objective is continuous.

There is a sense in which optima are uninteresting if they always satisfy $S^{p}(i) \equiv \mathbb{S}_{+}$. As our preview discussion suggests, that does not always happen. 
The following proposition says that if there is sufficient persistence, then the optimum has no trade whenever the money supply increases.

Proposition 3. Let $\left\{\Pi_{k}\right\} \rightarrow I$ (the identity matrix) and be such that the corresponding sequence of invariant probability vectors $\left\{p_{k}\right\}$ converges to a strictly positive vector. Assume that the optimum for any $\Pi$ in the neighborhood of $\Pi=I$ satisfies $u\left(\beta \min _{s} \Delta_{s}\right) \geq y_{\max }^{1}$. There exists $K$ such that if $k>K$, then the optimum for $\Pi_{k}$ has no-trade between informed producers and uninformed consumers whenever the current state, $j$, exceeds the previous state, $i$.

The optimum we have described is easy to implement, and without using the fiction of a computer at each meeting that receives reports and is updated when the previous state is publicly announced. At each trade meeting and conditional on the knowledge that is common to the two people in the meeting, the optimum has a single positive trade proposal. Indeed, this is a feature of any incentive-feasible mechanism. Therefore, we can let the two people play a simultaneous move game in which the strategies are simply \{trade, no-trade\}. If both say trade, then the positive trade proposal is carried out. If either says no-trade, then there is no trade and each goes on to the next date. In part because the optimum has positive trade in every state when the consumer is informed and the producer is not, it is a dominant strategy for the consumer in every trade meeting to play trade. Given that play by the consumer, it is a best response for the producer to play the strategy that implements the optimum.

Finally, we provide a sufficient condition for assuring that any optimum satisfies $u\left(\beta \min _{s} \Delta_{s}\right) \geq y_{\max }^{1}$, the hypothesis of Propositions 1-3. The sufficient condition uses a lower bound on the optimal magnitude of $Z$, a bound we call $Z_{\min }$. As part of the proof that shows that a small enough range for the support of the money supply is sufficient to ensure $u\left(\beta \min _{s} \Delta_{s}\right) \geq y_{\max }^{1}$ (lemma 6 below), we provide one such $Z_{\min }$. More generally, $Z_{\min }$ could be obtained from any lemma 3 mechanism that is incentive feasible.

We begin by constructing an upper bound on ex ante utility implied by any allocation that violates $u\left(\beta \min _{s} \Delta_{s}\right) \geq y_{\max }^{1}$. The idea is that violating this inequality implies that at least one component of $\beta \Delta$ is less than $u^{-1}\left(y_{\max }^{1}\right)$ and that this implies upper bounds on outputs and, hence, on ex ante utility. For each $k \in \mathbb{S}_{+}$, let $\Delta^{k} \in R^{S}$ be defined by $\Delta_{j}^{k}=y_{\max }^{1} / \beta$ for $j \neq k$ and $\beta \Delta_{k}^{k}=u^{-1}\left(y_{\max }^{1}\right)$. Then let $y^{k}$ be defined by

$$
\begin{aligned}
y^{k}(j, 0, i) & =\frac{\sum_{s=1}^{S} \pi_{i s}\left(\lambda-\theta_{s i}\right) \beta \Delta_{s}^{k}}{\sum_{s=1}^{S} \pi_{i s}\left(\lambda-\theta_{s i}\right)}, \\
y^{k}(j, j, i) & =y^{k}(0, j, i)=\beta \Delta_{j}^{k}, y^{k}(0,0, i)=\beta \sum_{s=1}^{S} \pi_{i s} \Delta_{s}^{k},
\end{aligned}
$$


for all $i, j \in \mathbb{S}_{+}$. The following lemma says that if the ex ante utility implied by $y^{k}$ is less than that implied by some incentive-feasible mechanism, then the optimum satisfies $u\left(\beta \min _{s} \Delta_{s}\right) \geq y_{\max }^{1}$.

Lemma 5. Let $Z\left(y^{k}\right)$ be ex ante welfare implied by $y^{k}$ as given by (5.5). Let $Z_{\min }$ be the ex ante welfare implied by some incentive-feasible mechanism. If there exists $Z_{\min }$ such that $Z\left(y^{k}\right) \leq Z_{\min }$ for each $k \in \mathbf{S}_{+}$, then any optimum satisfies $u\left(\beta \min _{s} \Delta_{s}\right) \geq y_{\max }^{1}$.

Although lemma 5 is a crude result because the specification in (5.5) is a crude upper bound on an incentive feasible $y$ given that $\beta \Delta_{k}<u^{-1}\left(y_{\max }^{1}\right)$ for some $k$, the next result says that its hypothesis can always be met if the range of the support for the money supply is sufficiently small.

Lemma 6. There exists $\varepsilon>0$ such that if $m_{S}-m_{1}<\varepsilon$, then the hypothesis of lemma 5 holds.

To summarize, we can determine in two steps whether a given environment satisfies conditions sufficient to ensure that any optimum satisfies $y_{\max }^{1} \leq y^{*}$ and $u\left(\beta \min \Delta_{i}\right) \geq y_{\max }^{1}$. First, we compute $y_{\max }^{1}$ and $y^{*}$ and check whether $y_{\max }^{1} \leq y^{*}$. (Then lemma 2 implies that any optimum satisfies $y_{\max }^{1} \geq \beta \max \Delta_{i}$.) Second, we attempt to find some $Z_{\text {min }}$ (the optimum from lemma 3 and the construction in the proof of lemma 6 are possibilities) for which the hypothesis of lemma 5 holds.

\section{EXAMPLES}

We present three examples designed mainly to emphasize the consequences for aggregates of whether trade is shut down in some meetings between informed producers and uninformed consumers. As discussed in the introduction, we expect any such shutting down to affect the magnitude of the total output response to changes in the amount of money relative to what happens in the benchmark case of everyone informed $(\lambda=1)$.

The aggregates we study are total output and the price level. For each example, we present the optimal Markov process for each aggregate. Let $Y_{i j}$ and $P_{i j}$ denote total output and the price level, respectively, when the previous state is $i$ and the current state is $j$. For a given mechanism, total output is the appropriate sum of outputs over meetings:

$$
\begin{aligned}
N Y_{i j}= & m_{i}\left(1-m_{i}\right)(1-\lambda)^{2} y(0,0, i)+\left(\lambda-\theta_{j i}\right) \theta_{j i} y(j, j, i)+ \\
& \left(\lambda-\theta_{j i}\right)\left(1-m_{i}\right)(1-\lambda) y(j, 0, i)+m_{i} \theta_{j i}(1-\lambda) y(0, j, i) .
\end{aligned}
$$


We take $P_{i j}$ to be the total output deflator; namely, total nominal output, denoted $X_{i j}$, divided by $Y_{i j} .{ }^{14}$ Because one unit of money is traded in each meeting in which trade occurs, for a given mechanism total nominal output is a weighted sum of the number of trades. That is, $N X_{i j}$ is the right-hand side of $(6.1)$ with $y\left(s, s^{\prime}, i\right)$ replaced by $a\left(s, s^{\prime}, i\right)$. The pair $\left(Y_{i j}, P_{i j}\right)$ occurs with probability $p_{i} \pi_{i j}$, where, as above, $p_{i}$ denotes the invariant probability that the state is $i$. Therefore, the pair $\left(Y_{i j}, P_{i j}\right)$ and the exogenous probability $p_{i} \pi_{i j}$ for each $(i, j)$ completely describe the Markov process for total output and the price level.

The first two examples share all but one feature. Both have $S=2$ and a symmetric $\Pi$ with $\pi_{i i}=1-\varepsilon$. We let $u$ be the square-root functiona simple function that satisfies our general assumptions. For that choice of $u, y^{*}=\frac{1}{4}$. We also let $N=3$, the minimum consistent with no doublecoincidence meetings. For both examples, the support for the money supply is $\left(m_{1}, m_{2}\right)=\left(\frac{4}{9}, \frac{1}{2}\right)$. We choose $\beta$ so that $y_{\max }^{1}=y^{*}$, which gives $\beta=\frac{27}{32}$. The two examples differ regarding $\varepsilon$. In accord with Proposition 3 , we expect to have trade occur all the time if $\varepsilon$ is not too close to zero and we expect to have no-trade occur when $(i, j)=(1,2)$ if $\varepsilon$ is small enough. Example 1 has $\varepsilon=0.1$, which implies that trade always occurs. Example 2 has $\varepsilon=.0025$, which turns out to be small enough to get no-trade in meetings between informed producers and uninformed consumers when $(i, j)=(1,2) .{ }^{15}$ For each $\varepsilon$, we report results for three values of $\lambda: \lambda=\frac{1}{9}$, the minimum consistent with our assumptions; $\lambda=\frac{11}{20}$, the magnitude which maximizes the probability of meetings between informed producers and uninformed consumers; and $\lambda=1$, the benchmark.

Before we present the results for aggregates, we present for one case the meeting-specific outputs from which the aggregates are deduced. When everyone is informed, there is only one kind of meeting per date and the output in that meeting depends only on the current state. In example 1 when $\lambda=1$, $y(1,1, i)=.2270$ and $y(2,2, i)=.2160$. Because there is only one kind of meeting, the price level is simply the inverse of meeting-specific output. Notice that even in the low money supply state, output is less than $y_{\max }^{1}=\frac{1}{4}$. This happens for two reasons: the money supply may increase, which would reduce the probability of meeting someone without money, and the producer may be given money without producing. In all our examples, the persistence

\footnotetext{
${ }^{14}$ We must use a price index because, in general, the price of output depends on the kind of meeting. The price in a meeting is $1 / y\left(s, s^{\prime}, i\right)$ for $\mathrm{s}$ and $s^{\prime} \in \mathbb{S}$. Therefore, for given past and current states, the price varies with who in the meeting is informed. An outside observer collecting such prices must use an index to compute $a$ price level.

${ }^{15}$ The sufficient condition given in lemma 5 is satisfied for examples 1 and 2 . The value of $Z\left(y^{k}\right)$, which is the same for each $k$ by the symmetry of the example, never rises above .12. This is below the values of $Z$ shown in tables 3 and 4 below, the values for the optima, which, as noted above, can play the role of $Z_{\min }$ in lemma 5 .
} 
in the money supply process is sufficient to imply that $\Delta_{1}>\Delta_{2}$; moreover, when $\lambda=1$ the ordering for aggregate output is the same as that for meetingspecific output. This happens in part because our money supplies are near $\frac{1}{2}$, where the function $m(1-m)$ is flat.

Table 2. Meeting specific outputs for example 1 with $\lambda=\frac{1}{9}$.

\begin{tabular}{|c|c|c|c|c|c|c|}
\hline \multicolumn{2}{|c|}{$y(j, j, i)$} & \multicolumn{2}{c|}{$y(0,0, i)$} & \multicolumn{2}{c|}{$y(j, 0, i), j>0$} & $y(0, j, i), j>0$ \\
\hline$j=1$ & $j=2$ & $i=1$ & $i=2$ & $i=1$ & $i=2$ & \\
\hline .22559 & .21541 & .22458 & .21642 & .22365 & .21541 & .21541 \\
\hline
\end{tabular}

Table 2 contains meeting-specific outputs for example 1 for $\lambda=\frac{1}{9}$. The first two columns contain output in meetings between informed people. These differ from those in the benchmark because $\lambda$ affects $\Delta$. The next two columns contain output in meetings between uninformed people. Here, there is no dependence on the current state and the dependence on the previous state is weaker than the dependence on the current state for meetings between informed people because the Markov process gives rise to less two-period persistence than one-period persistence. The next two columns contain output in meetings between informed consumers and uninformed producers. Here, output does not depend on the current state because of the truth-telling requirement. Notice also that for each $i$ output is lower than when two uninformed people meet. This happens because uninformed producers who meet informed people with money place more weight than $\pi_{12}$ on the possibility that the money supply has increased. (In fact, in this example, when the money supply decreases, no informed person has money, so when an uninformed producer meets an informed consumer with money, the producer is able to deduce that the current state is state 2 which implies the same output level as for an informed producer.) The last column contains output in meetings between uninformed consumers and informed producers. Here output depends on neither the current state nor the previous state and is the same as in a meeting between informed people when the current state is the high money supply state.

Tables 3 and 4 contain detailed results for examples 1 and 2. In particular, they contain the Markov processes for total output and the price level. The rows labeled $\frac{X_{i i^{\prime}}-X_{i i}}{X_{i i}}$ contain percentage changes of switching states relative to not switching states, except when they pertain to $\Delta$. For $\Delta$, the entry is $\frac{\Delta_{2}-\Delta_{1}}{\Delta_{1}}$. Also, all the meeting-specific outputs can be computed from the reported $\Delta$ using the applicable conditional expectations as given in Proposition 1. 
Table 3. Example 1: $\varepsilon=\frac{1}{10}$

\begin{tabular}{|c|c|c|c|c|c|c|c|}
\hline$\lambda$ & $Z$ & J & $Y_{1 j}$ & $Y_{2 j}$ & $P_{1 j}$ & $P_{2 j}$ & $\Delta$ \\
\hline \multirow{3}{*}{1} & \multirow{3}{*}{132033.} & & .0186857 & .0186857 & 4.405 & 4.405 & .2691 \\
\hline & & 2 & .0180001. & .0180001. & 4.630 & 4.630 & .2560 \\
\hline & & & $-3.67 \%$ & $3.81 \%$ & $5.11 \%$ & $-4.86 \%$ & $-4.87 \%$ \\
\hline \multirow{3}{*}{$\frac{11}{20}$} & \multirow{3}{*}{.131924. } & & $18 \%$ & 01792 & .521 & 4.592 & .2654 \\
\hline & & 2 & 0182349. & .01792350 & 4.570 & 4.649 & .2545 \\
\hline & & & $0.15 \%$ & $-0.0008 \%$ & $1.09 \%$ & $-1.23 \%$ & $-4.13 \%$ \\
\hline \multirow{3}{*}{$\frac{1}{9}$} & \multirow{3}{*}{131988.} & & & & & 4.6249 & .2674 \\
\hline & & & .0186 & .018 & 4.4589 & 4.6251 & 2553 \\
\hline & & $\frac{X_{i i^{\prime}}-X_{i i}}{X .}$ & $1.56 \%$ & $-1.23 \%$ & $-0.30 \%$ & $-0.004 \%$ & $-4.52 \%$ \\
\hline
\end{tabular}

Table 4. Example 2: $\varepsilon=\frac{1}{400}$

\begin{tabular}{|c|c|c|c|c|c|c|c|}
\hline$\lambda$ & $Z$ & $j$ & $Y_{1 j}$ & $Y_{2 j}$ & $P_{1 j}$ & $P_{2 j}$ & $\Delta$ \\
\hline \multirow{3}{*}{1} & \multirow{3}{*}{.132325} & 1 & .0205136 & .0205136 & 4.012 & 4.012 & .29540 \\
& & 2 & .0186943 & .0186943 & 4.458 & 4.458 & .26587 \\
& & $\frac{X_{i i^{\prime}}-X_{i i}}{X_{i i}}$ & $-8.87 \%$ & $9.73 \%$ & $11.10 \%$ & $-9.99 \%$ & $-9.99 \%$ \\
\hline \multirow{2}{*}{$\frac{11}{20}$} & \multirow{3}{*}{.132291} & 1 & .0205003 & .0190627 & 4.015 & 4.318 & .29524 \\
& & 2 & .0159842 & .0186940 & 4.171 & 4.458 & .26584 \\
& & $\frac{X_{i i^{\prime}}-X_{i i}}{X_{i i}}$ & $-22.03 \%$ & $1.97 \%$ & $3.89 \%$ & $-3.14 \%$ & $-9.96 \%$ \\
\hline \multirow{3}{*}{$\frac{1}{9}$} & \multirow{3}{*}{.132324} & 2 & .0205071 & .0184667 & 4.013 & 4.45691 & .29538 \\
& & $\frac{X_{i i^{\prime}}-X_{i i}}{X_{i i}}$ & $0.23 \%$ & .0186975 & 4.015 & 4.45693 & .26586 \\
& & & $-1.23 \%$ & $0.03 \%$ & $-0.0003 \%$ & $-9.99 \%$ \\
\hline
\end{tabular}

As a way to highlight some of the impact effects, we present in Figures 2 the effects of a "persistent" increase in the amount of money on output and the price level. That is, we present aggregates corresponding to a sequence of states $(1,1,2,2)$ starting with the second term so that the abscissa label "1" in the figures is a date at which both the current and previous states are state 1. (While we could have presented the effects of an increase followed by a decrease, given the high degree of persistence in both examples, such an event is extremely rare.) As suggested above, impact effects on aggregate output depend on whether trade is ever shut down and on the probability of meetings between informed producers and uninformed consumers. For $\varepsilon=0.1$, for which there is always-trade, there are positive impact effects on output relative to the benchmark. (When the money supply increases, meeting-specific output is higher in meetings between informed consumers and uninformed producers than it is between informed people when $\lambda=1$.) For $\varepsilon=0.0025$, for which there is no-trade when informed producers meet uninformed consumers and the money supply has increased, the direction of effect depends on $\lambda$. When $\lambda=$ $\frac{11}{20}$, which maximizes the probability of meetings between informed producers 
and uninformed consumers, the increase produces a large decline in output relative to what happens in the benchmark. When $\lambda=\frac{1}{9}$, that does not happen because most informed people have money when the amount of money increases.

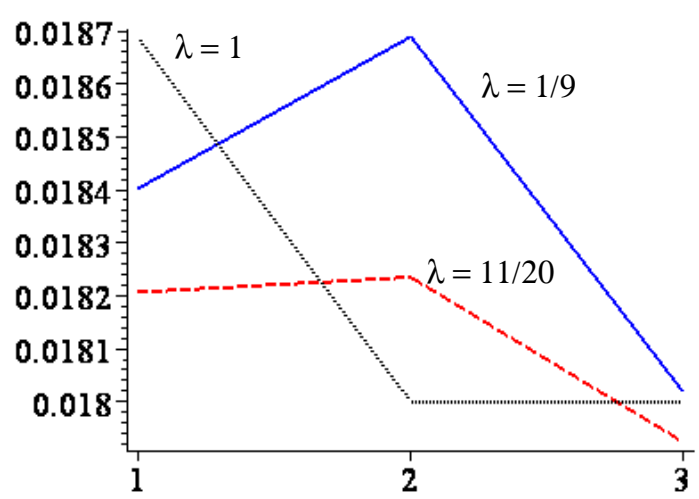

Figure 2a: Output, Example 1

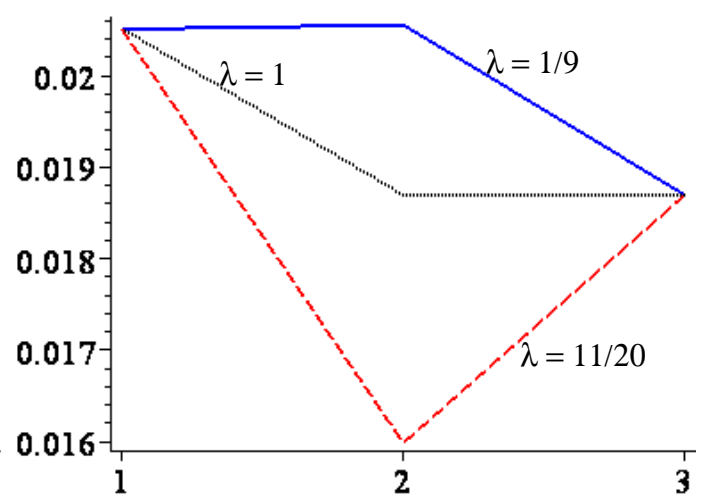

Figure 2b: Output, Example 2

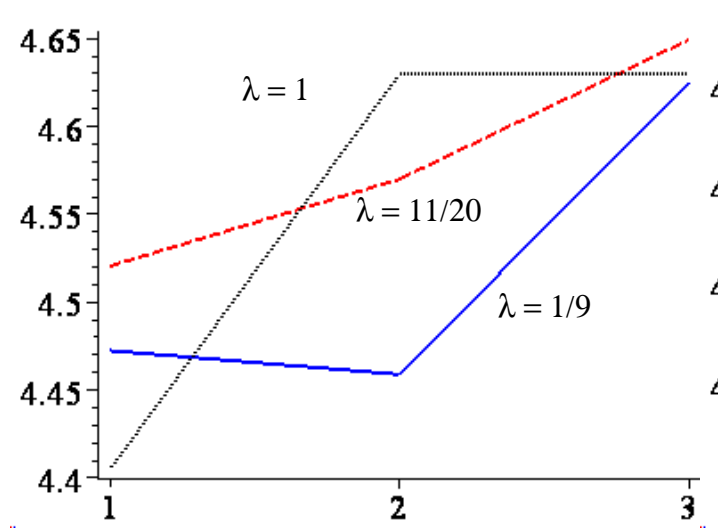

Figure 2c: Prices, Example 1

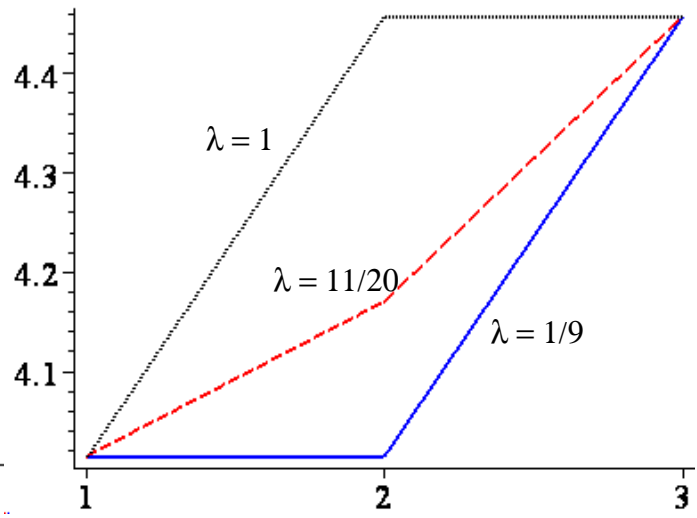

Figure 2d: Prices, Example 2

Recall that the price in each kind of meeting is simply the inverse of output in the meeting and that the only meeting-specific price that changes in example 1 when the money supply changes is that in meetings between the informed. That effect tends to make the (aggregate) price level change in the same direction as the money supply. But, because it is the only price that changes, the impact effects on the price level tend to be weaker the smaller is $\lambda$. Moreover, that effect can even be offset by compositional effects, which happens in example 1 when $\lambda=\frac{1}{9}$. When $\lambda=\frac{1}{9}$ and the money supply increases, only $\frac{1}{18}$ of the informed are without money. Hence, there are very few trade-meetings among 
the informed. Also, there are fewer meetings between informed producers and uninformed consumers and more meetings between informed consumers and uninformed producers. Under always-trade, the meeting-specific price is higher when producers are relatively informed than when consumers are relatively informed. Hence, the altered composition produced by the monetary increase tends to lower the price index.

In these examples the highest level of ex ante welfare, $Z$, is achieved when everyone is informed. However, ex ante welfare is not monotone in $\lambda$. The prevalence of meetings between informed producers and uninformed consumers, which depends on $\lambda$ in a non-monotone way, is one source of the non-monotonicity of welfare. In example 1, when there is always-trade, output is minimal in such meetings because it satisfies the producer's individual rationality constraint for the smaller component of $\Delta$ no matter what is the current state. In example 2 , this low output is partly overcome, but only at the cost of shutting down trade when the money supply increases.

Example 3 is designed to have non-monotone impact effects of increases in the amount of money on output. The example has $S=3$ and combines features of examples 1 and 2 so that large increases in the amount of money produce a shutting down of trade, but small increases do not. We continue to let $u$ be the square-root function, and let $N=3$. The support for the money supply is $\left(m_{1}, m_{2}, m_{3}\right)=\left(\frac{5}{12}, \frac{4}{9}, \frac{1}{2}\right) \approx(.4167, .4444, .5000)$, while $\beta=\frac{36}{43}$ so that, again, $y_{\max }^{1}=y^{*}$. For $\Pi$, we choose,

$$
\Pi=\left[\begin{array}{ccc}
1-\varepsilon_{1}-\varepsilon_{2} & \varepsilon_{1} & \varepsilon_{2} \\
\varepsilon_{1} & 1-2 \varepsilon_{1} & \varepsilon_{1} \\
\varepsilon_{2} & \varepsilon_{1} & 1-\varepsilon_{1}-\varepsilon_{2}
\end{array}\right]
$$

with $\left(\varepsilon_{1}, \varepsilon_{2}\right)=(.0500, .0025)$. Even though the range for the money supply is larger for this example, it remains true that the hypothesis of lemma 5 holds. ${ }^{16}$ For this example, we computed results only for $\lambda=1$ and $\lambda=\frac{11}{20}$.

\footnotetext{
${ }^{16}$ This is shown by comparing the value of $Z\left(y^{k}\right)=.1104$ (which is the same for each $k$, by the symmetry of the example) with the value of $Z$ in the table; note that $\Delta_{1}>\Delta_{2}>\Delta_{3}$, so that the mechanism that sets $a(0,3,1)=0$ satisfies producer truth-telling. Also, consumer individual rationality constraints hold because $u\left(\beta \Delta_{3}\right)>\beta \Delta_{1}$. Therefore, the value of $Z$ is a valid choice for $Z_{\min }$.
} 
Table 5. Example 3: $\left(\varepsilon_{1}, \varepsilon_{2}\right)=\left(\frac{1}{20}, \frac{1}{400}\right)$

\begin{tabular}{|c|c|c|c|c|c|c|c|c|c|}
\hline$\lambda$ & $Z$ & ${ }_{j}^{j}$ & $\overline{Y_{1 j}}$ & $\overline{Y_{2 j}}$ & $Y_{3 j}$ & $P_{1 j}$ & $P_{2 j}$ & $P_{3 j}$ & $\Delta$ \\
\hline \multirow{3}{*}{1} & \multirow{3}{*}{1259} & 1 & .01953 & .01953 & .01953 & 4.148 & 4.148 & 4.148 & 2879 \\
\hline & & 2 & 01858. & .01858 & 01858 & 4.430 & 4.430 & 4.430 & .2696 \\
\hline & & 3 & 01751 & 01751 & 01751 & 4.759 & 4.759 & 4.759 & 2510 \\
\hline \multirow{3}{*}{$\frac{11}{20}$} & \multirow{3}{*}{.1257. } & 1 & .01874 & .01797 & .01755 & 4.324 & 4.508 & 4.617 & 2810 \\
\hline & & 2 & .01870. & 01793 & 01750 & 4.401 & 4.589 & 4.702 & 2638 \\
\hline & & 3 & .01542. & .01791. & 01743 & 4.441 & 4.654 & 4.781 & 2495 \\
\hline
\end{tabular}

As in the first two examples, in the benchmark total output is decreasing in the amount of money. When $\lambda=\frac{11}{20}$, small increases in the amount of money are expansionary relative to the benchmark. That is, $Y_{12} / Y_{11}$ and $Y_{23} / Y_{22}$ are each larger than their values for the benchmark. However, as promised, a large increase is contractionary relative to the benchmark. That is, $Y_{13} / Y_{11}$ is smaller than its value in the benchmark. As in the other examples, the price level is less responsive to changes in the amount of money than it is in the benchmark. Again, welfare is lower with uninformed people than when everyone is informed.

\section{RANDOMIZATION}

The mechanisms studied above are deterministic. More general mechanisms would permit randomization. There are three conceivable kinds of randomization. First, there could be randomization over outcomes, some of which include positive production and no transfer of money from the consumer to the producer. Such randomization would not satisfy our individual-rationality constraints, because the producer would not accept an outcome that calls for production and no transfer of money. ${ }^{17}$ Second, there could be randomization over outcomes in which each of several different amounts of production might be exchanged for a unit of money. While such randomization could be incentive feasible, we doubt that it could be optimal. We suspect that shifting probability toward the highest output that occurs with positive probability would be incentive feasible and would raise ex ante welfare. Third, there could be randomization over trade and no-trade sets. This kind of randomization is also incentive feasible and, as we show by example, can be optimal. We first present the example and then comment more generally on such randomization.

The reasoning behind the example is as follows. Consider the symmetric $\Pi$ used in the $S=2$ examples. By Proposition 3 and our examples, there is a critical value of $\varepsilon$ at which the optimum switches from $S^{p}(1)=\{1\}$, trade is shut down when the money supply increases, to $S^{p}(1)=\{1,2\}$, always-trade.

\footnotetext{
${ }^{17}$ See [2] for the study of such randomization in a similar model without uncertainty, but in which it is assumed that people can commit to the outcome of randomization.
} 
At that point, the mechanism varies discontinuously, but $Z$ does not. Randomization over whether trade occurs could smooth the mechanism and, as we now explain, is likely to raise $Z$. For $q \in[0,1]$, let the pair $[a(0,2,1), y(0,2,1)]$ be specified as $\left[1, \beta \Delta_{2}\right]$ with probability $q$, and $[0,0]$ with probability $1-q$, and let $y(0,2,1)=\beta \Delta_{2}$, and $y(0,1,1)=(1-q) \beta \Delta_{1}+q \beta \Delta_{2}$. For $q=0$, this corresponds to $S^{p}(1)=\{1\}$, while for $q=1$, it corresponds to $S^{p}(1)=\{1,2\}$. Also, if $\Delta_{1} \geq \Delta_{2}$, then it satisfies truth-telling for the informed producer. The part of $Z$ that depends directly on $q$ is proportional to

$$
(1-\varepsilon) \theta_{11} z\left((1-q) \beta \Delta_{1}+q \beta \Delta_{2}\right)+\varepsilon \theta_{21} q z\left(\beta \Delta_{2}\right) .
$$

Holding $\Delta$ fixed, this term is strictly concave in $q$. Therefore, if this term has the same value at $q=0$ and at $q=1$ - which, by Proposition 2 , happens at the critical value of $\varepsilon$ - then it takes higher values at interior values of $q$. Although suggestive, this conclusion is not decisive because it ignores the dependence of $\Delta$ on $q$. Therefore, we produce an example which takes this dependence into account.

The example is the same as the $S=2$ examples in section 6 , except that $\lambda=\frac{1}{2}$ and $\varepsilon=\frac{1}{200}$. This $\varepsilon$ is very near the critical one at which the optimum switches from $S^{p}(1)=\{1\}$ to $S^{p}(1)=\{1,2\}$. Letting $Z(q)$ denote ex ante welfare as a function of $q$, we find $Z(1)=.1322575, Z(0)=.1322572$, and $Z\left(\frac{1}{2}\right)=.1322645$. Therefore, always-trade, $q=1$, is slightly better than shutting down trade, $q=0$, but mixing with $q=\frac{1}{2}$ is better still. Thus randomization is optimal in this example.

Despite this finding, we suspect that randomization would not drastically change the kind of necessary conditions we found and, therefore, the kind of possibilities for the optimum that we displayed in section 6 . Proposition 1 identifies a finite set $\mathcal{M}$ of mechanisms that contains the optimum (where each mechanism in $\mathcal{M}$ corresponds to a choice of trade sets $S^{p}$ ). The welfare function $Z$ gives an ordering of $\mathcal{M}$. We conjecture that the optimal randomized mechanism can be interpreted as a point in the convex hull of $\mathcal{M}$, supported by a subset of mechanisms in $\mathcal{M}$ that are close (in terms of welfare) to the optimal deterministic mechanism.

\section{TAKE-IT-OR-LEAVE-IT OFFERS BY CONSUMERS}

Matching models of the sort we are studying have often been analyzed using a bargaining rule. Among the most commonly used, because it is simple, is take-it-or-leave-it offers by consumers: trade occurs if and only if the producer agrees to supply some amount $x$, chosen by the consumer. Our optimum resembles an equilibrium under such bargaining because, according to Proposition 1, producers are being pushed to where they are on the margin between accepting and rejecting trade. In fact, any equilibrium under deterministic take-it-or-leave-it offers by consumers is one of the candidates for an optimum, 
one of the mechanisms that satisfy Proposition 1. However, the optimum is not always such an equilibrium. In particular, at an optimum, an uninformed consumer facing an informed producer and making a take-it-or-leave-it offer leans toward an $x$ that shuts down trade in more states than the optimum dictates. $^{18}$

This is easy to see if, for the moment, we ignore the dependence of $\Delta$ on the $S^{p}(i)$ sets. Then, if $\Delta_{j}>\Delta_{j+1}$, the ex ante welfare gain from having positive output in one additional state, $r$, when the previous state is $i$ is proportional to

$$
z\left(\beta \Delta_{r}\right) \sum_{j=1}^{r} \pi_{i j} \theta_{j i}-z\left(\beta \Delta_{r-1}\right) \sum_{j=1}^{r-1} \pi_{i j} \theta_{j i} \equiv B_{p}(r, i) .
$$

The difference here represents a trade-off between the output $\beta \Delta_{r-1}$ with some probability and the lower output $\beta \Delta_{r}$ with a higher probability. In contrast, the net gain to the consumer from demanding the smaller output $\beta \Delta_{r}$ rather than the output $\beta \Delta_{r-1}$ is proportional to

$$
\sum_{j=1}^{r} \pi_{i j} \theta_{j i}\left[u\left(\beta \Delta_{r}\right)-\beta \Delta_{j}\right]-\sum_{j=1}^{r-1} \pi_{i j} \theta_{j i}\left[u\left(\beta \Delta_{r-1}\right)-\beta \Delta_{j}\right]=B_{p}(r, i)-\beta\left(\Delta_{r-1}-\Delta_{r}\right) \sum_{j=1}^{r-1} \pi_{i j} \theta_{j i}
$$

Thus, the consumer sees an additional cost to having output in one additional state; namely, that the consumer surrenders money for less than its value (conceding an informational rent to the producer). But this cost is not part of ex ante welfare because the distribution of money at the next date is unaffected by whether trade occurs.

Of course, the above comparison is not the correct one because it ignores the dependence of $\Delta$ on the $S^{p}(i)$ sets. Nevertheless, the comparison correctly suggests that if the optimum has always-trade by a small enough margin, then the optimum is not an equilibrium under take-it-or-leave-it offers by consumers That happens for the example presented in the discussion of randomization. There the optimum has always-trade and implies $\left(\Delta_{1}, \Delta_{2}\right)=$ $(.2855514, .265532)$. If the uninformed consumer who meets an informed producer demands $\beta \Delta_{1}$ in exchange for surrendering money, then the consumer's expected gain is $\frac{199}{200}\left[\left(\beta \Delta_{1}\right)^{\frac{1}{2}}-\beta \Delta_{1}\right]$, where, recall, $\beta=\frac{27}{32}$. If, instead, the consumer demands $\beta \Delta_{2}$ in exchange for surrendering money, then the expected gain is $\left(\beta \Delta_{2}\right)^{\frac{1}{2}}-\frac{199}{200} \beta \Delta_{1}-\frac{1}{200} \beta \Delta_{2}$. The difference between the former and the latter is $\frac{199}{200}\left(\beta \Delta_{1}\right)^{\frac{1}{2}}-\left(\beta \Delta_{2}\right)^{\frac{1}{2}}+\frac{1}{200} \beta \Delta_{2}$, which is positive. Therefore, in this

\footnotetext{
${ }^{18}$ The only reason the two may coincide is because we have imposed restrictions to keep output less than $y^{*}$. Someone who simply assumes take-it-or-leave-it bargaining would not impose those restrictions.
} 
case, the always-trade optimum is not an equilibrium under take-it-or-leave-it offers by consumers. ${ }^{19}$

\section{Concluding Remarks}

We have worked with a minimal model and a minimal class of mechanisms. In addition to considering randomization and other regions of the parameter space, it would be desirable to check the robustness of our findings to some of our more extreme assumptions. We have made substantial use of the indivisibility of money and the unit upper bound. Obviously, truth-telling constraints would not have such simple implications if the money trades could be other than surrendering 0 or 1 unit. Settings with richer individual holdings of money would be much harder to study because in them the distribution of money holdings depends on the mechanism. Another extension to consider is an information structure in which the identity of the informed is not common knowledge. Such a change gives rise to additional truth-telling constraints and to two-sided differential information in meetings. A third extension would consider lengthening the lag with which there is a public announcement of the state. Such a version would permit the study of whether it is optimal for relatively informed people to tell their trading partners what they know as they leave meetings.

In an important sense, though, the simplicity of our model is a virtue. Our background environment was designed by others to depict absence-ofdouble-coincidence problems so severe that the use of money is the only way to depart from autarky. We have put into that environment monetary uncertainty and differential information about realizations in a way that does not tie a person's information status to the person's money holdings. Then, despite assuming that money holdings are either 0 or 1 unit, that public revelation of realizations occurs after one period, that there is common knowledge about who is informed, and that the society finds the best way of conducting trade in the presence of the uncertainty and the implied differential information, we find that the model delivers a rich theory of output and price level responses.

Our theory is special in that for tractability we have assumed an arbitrary restiction on money holdings. To get around this, we compare results of the differential information model with the analogous results in a benchmark model in which everyone is informed. This comparison shows that the price level tends to be sticky and there can be positive or negative impact effects on

\footnotetext{
${ }^{19}$ As might be suspected, an equilibrium under take-it-or-leave-it offers by consumers may not exist unless there is randomization when the consumer is indifferent between two offers. In the above example, the $S^{p}(1)=\{1\}$ mechanism that satisfies proposition 1 is an equilibrium under take-it-or-leave-it offers by consumers. This is confirmed by showing that $\frac{199}{200}\left(\beta \Delta_{1}\right)^{\frac{1}{2}}-\left(\beta \Delta_{2}\right)^{\frac{1}{2}}+\frac{1}{200} \beta \Delta_{2} \geq 0$ when evaluated at the $\Delta$ implied by that mechanismnamely, $\left(\Delta_{1}, \Delta_{2}\right)=(.294211, .265728)$.
} 
output - although negative impact effects turn up only for very low probability increases in the money supply. Even if everything is kept symmetric, there is a tendency for money and output to be positively related, but in some unusual contingencies the relationship goes the other way. Moreover, even in the case of a positive relationship, the effects of increases and decreases in the quantity of money are not symmetric. We conjecture that similar possibilities arising from differential information would be present in a model with less restrictive money holdings.

\section{Appendix: Proofs}

To begin, we express the dependence of the $V_{k}$ on the mechanism. It is convenient to do this in two steps. We let $v_{k}(\iota, s, i):\{0,1\} \times \mathbb{S}_{+} \times \mathbb{S}_{+} \rightarrow R_{+}$ denote the expected discounted utility of someone who (i) holds $k \in\{0,1\}$ units of money, and (ii) is either informed $(\iota=1)$ or uninformed $(\iota=0)$ when the current state is $s$ and the previous state is $i$-all of this prior to meetings but after determination of the set of newly informed people and after additions or subtractions of money. These continuation values satisfy

$$
\begin{aligned}
& N v_{0}(\iota, j, i)=N \beta V_{0}(j)+\left(\lambda-\theta_{j i}\right) G_{0}(1, \iota, j, i)+(1-\lambda) m_{i} G_{0}(0, \iota, j, i) \\
& N v_{1}(\iota, j, i)=N \beta V_{1}(j)+\theta_{j i} G_{1}(\iota, 1, j, i)+(1-\lambda)\left(1-m_{i}\right) G_{1}(\iota, 0, j, i) .
\end{aligned}
$$

In terms of them, the $V_{k}(i)$ are defined by

$$
\begin{aligned}
& V_{0}(i)=\sum_{j=1}^{S} \pi_{i j}\left[(1-\lambda) v_{0}(0, j, i)+\lambda v_{0}(1, j, i)\right]+\sum_{j=i}^{S} \pi_{i j} \eta_{i j}\left(v_{1}(1, j, i)-v_{0}(1, j, i)\right) \\
& V_{1}(i)=\sum_{j=1}^{S} \pi_{i j}\left[(1-\lambda) v_{1}(0, j, i)+\lambda v_{1}(1, j, i)\right]-\sum_{j=1}^{i-1} \pi_{i j} \eta_{i j}\left(v_{1}(1, j, i)-v_{0}(1, j, i)\right),
\end{aligned}
$$

where

$$
\eta_{i j}=\left\{\begin{array}{l}
1-\frac{m_{j}}{m_{i}} \text { if } j<i \\
1-\frac{1-m_{j}}{1-m_{i}} \text { if } j \geq i
\end{array} .\right.
$$

It follows from the the lower bound on $\lambda$ that $\eta_{i j} \leq \lambda$. Equations (10.2) with the $v_{k}(\iota, s, i)$ replaced by their expressions in (10.1) consist of $2 S$ equations in $V_{0}$ and $V_{1}$ that are linear for a given $(y, a)$. Moreover, with that substitution, for a given $(y, a)$ the right sides of equations (10.2) can be viewed as a mapping from $R^{2 S}$ to $R^{2 S}$. It is immediate that that mapping satisfies Blackwell's sufficient conditions for contraction: monotonicity and discounting. Therefore, 
equations (10.2) have a unique solution for $V_{0}$ and $V_{1}$ for a given $(y, a)$. The implied unique $\Delta \equiv V_{1}-V_{0}$ is denoted $\Delta(y, a)$.

Lemma 1. For any mechanism, $Z$ satisfies (4.13).

Proof. From the definition of $\eta_{i j}$ above,

$$
m_{j}-m_{i}=\left\{\begin{array}{l}
-m_{i} \eta_{i j} \text { if } j<i \\
\left(1-m_{i}\right) \eta_{i j} \text { if } j \geq i
\end{array} .\right.
$$

Then, if we substitute (10.2) into (4.12), we obtain

$$
\begin{aligned}
Z= & \sum_{i=1}^{S} p_{i}\left[m_{i} V_{1}(i)+\left(1-m_{i}\right) V_{0}(i)\right] \\
= & \sum_{i=1}^{S} \sum_{j=1}^{S} p_{i} \pi_{i j}(1-\lambda)\left[m_{i} v_{1}(0, j, i)+\left(1-m_{i}\right) v_{0}(0, j, i)\right] \\
& +\sum_{i=1}^{S} \sum_{j=1}^{S} p_{i} \pi_{i j} \lambda\left[m_{i} v_{1}(1, j, i)+\left(1-m_{i}\right) v_{0}(1, j, i)\right] \\
& +\sum_{i=1}^{S} \sum_{j=1}^{S} p_{i} \pi_{i j}\left(m_{j}-m_{i}\right)\left[v_{1}(1, j, i)-v_{0}(1, j, i)\right]
\end{aligned}
$$

Therefore,

$$
\begin{aligned}
Z= & \sum_{i=1}^{S} \sum_{j=1}^{S} p_{i} \pi_{i j}(1-\lambda)\left[m_{i} v_{1}(0, j, i)+\left(1-m_{i}\right) v_{0}(0, j, i)\right] \\
& +\sum_{i=1}^{S} \sum_{j=1}^{S} p_{i} \pi_{i j}\left[\left(\lambda-\theta_{j i}\right) v_{1}(1, j, i)+\theta_{j i} v_{0}(1, j, i)\right] .
\end{aligned}
$$


Using (10.1), we have

$$
\begin{aligned}
Z= & \beta \sum_{i=1}^{S} \sum_{j=1}^{S} p_{i} \pi_{i j}(1-\lambda)\left[m_{i} V_{1}(j)+\left(1-m_{i}\right) V_{0}(j)\right] \\
& +\beta \sum_{i=1}^{S} \sum_{j=1}^{S} p_{i} \pi_{i j}\left[\left(\lambda-\theta_{j i}\right) V_{1}(j)+\theta_{j i} V_{0}(j)\right] \\
& +\frac{1}{N} \sum_{i=1}^{S} \sum_{j=1}^{S} p_{i} \pi_{i j}(1-\lambda) m_{i}\left[\theta_{j i} G_{1}(0,1, j, i)+(1-\lambda)\left(1-m_{i}\right) G_{1}(0,0, j, i)\right] \\
& +\frac{1}{N} \sum_{i=1}^{S} \sum_{j=1}^{S} p_{i} \pi_{i j}(1-\lambda)\left(1-m_{i}\right)\left[\left(\lambda-\theta_{j i}\right) G_{0}(1,0, j, i)+(1-\lambda) m_{i} G_{0}(0,0, j, i)\right] \\
& +\frac{1}{N} \sum_{i=1}^{S} \sum_{j=1}^{S} p_{i} \pi_{i j}\left(\lambda-\theta_{j i}\right)\left[\theta_{j i} G_{1}(1,1, j, i)+(1-\lambda)\left(1-m_{i}\right) G_{1}(1,0, j, i)\right] \\
& +\frac{1}{N} \sum_{i=1}^{S} \sum_{j=1}^{S} p_{i} \pi_{i j} \theta_{j i}\left[\left(\lambda-\theta_{j i}\right) G_{0}(1,1, j, i)+(1-\lambda) m_{i} G_{0}(0,1, j, i)\right] .
\end{aligned}
$$

Then, using (4.2), this expression can be written as

$$
\begin{aligned}
Z= & \beta \sum_{i=1}^{S} \sum_{j=1}^{S} p_{i} \pi_{i j}\left[m_{j} V_{1}(j)+\left(1-m_{j}\right) V_{0}(j)\right]+ \\
& \frac{1}{N} \sum_{i=1}^{S} \sum_{j=1}^{S} p_{i} \pi_{i j}(1-\lambda) m_{i}\left[\theta_{j i} z(y(0, j, i))+(1-\lambda)\left(1-m_{i}\right) z(y(0,0, i))\right]+ \\
& \frac{1}{N} \sum_{i=1}^{S} \sum_{j=1}^{S} p_{i} \pi_{i j}\left(\lambda-\theta_{j i}\right)\left[\theta_{j i} z(y(j, j, i))+(1-\lambda)\left(1-m_{i}\right) z(y(j, 0, i))\right] .
\end{aligned}
$$

Finally, using the definition of the invariant probabilities, the $p_{i}$,

$$
\begin{gathered}
\sum_{i=1}^{S} \sum_{j=1}^{S} p_{i} \pi_{i j}\left[m_{j} V_{1}(j)+\left(1-m_{j}\right) V_{0}(j)\right]= \\
\sum_{j=1}^{S}\left[m_{j} V_{1}(j)+\left(1-m_{j}\right) V_{0}(j)\right] \sum_{i=1}^{S} p_{i} \pi_{i j}= \\
\sum_{j=1}^{S} p_{j}\left[m_{j} V_{1}(j)+\left(1-m_{j}\right) V_{0}(j)\right]=Z .
\end{gathered}
$$


When this is substituted into (10.7), we get (4.13), as required.

We next establish some facts about $\Delta$. The first result is about the weighted average of $\Delta$ that appears in the IR constraint for uninformed producers in meetings with informed consumers, (4.10).

Lemma 7. If $(y, a)$ is incentive feasible, then $\Delta(y, a)$ satisfies,

$$
\frac{1}{\gamma_{i}^{1}\left(S^{c}(i)\right)} \sum_{j \in S^{c}(i)} \pi_{i j}\left(\lambda-\theta_{j i}\right) \beta \Delta_{j} \leq \frac{1}{\gamma_{i}^{1}\left(\mathbb{S}_{+}\right)} \sum_{j \in \mathbb{S}_{+}} \pi_{i j}\left(\lambda-\theta_{j i}\right) \beta \Delta_{j},
$$

where $\gamma_{i}^{1}\left(S^{c}(i) \equiv \sum_{j \in S^{c}(i)} \pi_{i j}\left(\lambda-\theta_{j i}\right)\right.$

Proof. We have

$$
\begin{aligned}
D & \equiv \frac{\beta}{\gamma_{i}^{1}\left(\mathbb{S}_{+}\right)} \sum_{j \in \mathbb{S}_{+}} \pi_{i j}\left(\lambda-\theta_{j i}\right) \Delta_{j}-\frac{\beta}{\gamma_{i}^{1}\left(S^{c}(i)\right)} \sum_{j \in S^{c}(i)} \pi_{i j}\left(\lambda-\theta_{j i}\right) \Delta_{j} \\
& =\frac{\beta\left(\sigma-\sigma^{\prime}\right)}{\sigma \sigma^{\prime}} \sum_{j \in S^{c}(i)} \pi_{i j}\left(\lambda-\theta_{j i}\right) \Delta_{j}+\frac{\beta}{\sigma^{\prime}} \sum_{j \in \mathbf{S}_{+}-S^{c}(i)} \pi_{i j}\left(\lambda-\theta_{j i}\right) \Delta_{j},
\end{aligned}
$$

where $\sigma \equiv \gamma_{i}^{1}\left(S^{c}(i)\right) \leq \sigma^{\prime} \equiv \gamma_{i}^{1}\left(\mathbb{S}_{+}\right)$. Now, let $x=\min _{j \in \mathbb{S}_{+}-S^{c}(i)} \Delta_{j}$. Then, by $(4.9)$,

$$
D \geq \frac{\beta\left(\sigma-\sigma^{\prime}\right)}{\sigma^{\prime}} x+\frac{\beta}{\sigma^{\prime}}\left(\sigma^{\prime}-\sigma\right) x=0
$$

as required.

The next lemma describes some properties of a mapping whose fixed point is $\Delta(y, a)$. Let

$$
h(x ; y, a) \equiv b(y)+\beta C(a) x \text { for } x \in R^{S} .
$$

where the $S \times S$ matrix $C(a) \equiv\left[c_{i j}(a)\right]$ and the $S \times 1$ vector $b(y) \equiv\left[b_{i}(y)\right]$ are defined as

$$
\begin{gathered}
\frac{c_{i j}(a)}{\pi_{i j}}=(1-\lambda)\left[1-\frac{1-\lambda}{N} a(0,0, i)-\frac{\theta_{j i}}{N} a(0, j, i)-\frac{\lambda-\theta_{j i}}{N} a(j, 0, i)\right]+ \\
\left(\lambda-\eta_{i j}\right)\left\{1-\frac{\lambda}{N} a(j, j, i)-\frac{(1-\lambda)}{N}\left[\left(1-m_{i}\right) a(j, 0, i)+m_{i} a(0, j, i)\right]\right\}
\end{gathered}
$$


and

$$
\begin{aligned}
N b_{i}(y)= & (1-\lambda)^{2}\left[\left(1-m_{i}\right) u(y(0,0, i))+m_{i} y(0,0, i)\right]+ \\
& (1-\lambda) \sum_{j=1}^{S} \pi_{i j}\left[\theta_{j i} u(y(0, j, i))+\left(\lambda-\theta_{j i}\right) y(j, 0, i)\right]+ \\
& \sum_{j=1}^{S} \pi_{i j}\left(\lambda-\eta_{i j}\right)\left[\theta_{j i} u(y(j, j, i))+\left(\lambda-\theta_{j i}\right) y(j, j, i)\right]+ \\
& (1-\lambda) \sum_{j=1}^{S} \pi_{i j}\left(\lambda-\eta_{i j}\right)\left[\left(1-m_{i}\right) u(y(j, 0, i))+m_{i} y(0, j, i)\right] .
\end{aligned}
$$

Lemma 8. Let $h(x ; y, a)$ be given by (10.12)-(10.14). Then (i) $\Delta(y, a)$ is a fixed point of $h(\cdot ; y, a)$; (ii) $h(\cdot ; y, a)$ satisfies Blackwell's sufficient conditions for contraction; (iii) if $h\left(\Delta(y, a) ; y^{\prime}, a^{\prime}\right) \geq \Delta(y, a)$, then $\Delta\left(y^{\prime}, a^{\prime}\right) \geq \Delta(y, a)$; and (iv) if $y^{\prime} \geq y$, then $\Delta\left(y^{\prime}, a\right) \geq \Delta(y, a)$.

Proof. From (10.2), we have

$$
\Delta_{i}=\sum_{j=1}^{S} \pi_{i j}\left[(1-\lambda) \delta_{i}(0, j)+\left(\lambda-\eta_{i j}\right) \delta_{i}(1, j)\right],
$$

where $\delta_{i}(\iota, j) \equiv v_{1}(\iota, j, i)-v_{0}(\iota, j, i)$. Also, from (10.1), we have

$$
\begin{aligned}
N \delta_{i}(\iota, j)= & N \beta \Delta_{j}+\theta_{j i} G_{1}(\iota, 1, j, i)+(1-\lambda)\left(1-m_{i}\right) G_{1}(\iota, 0, j, i) \\
& -\left(\lambda-\theta_{j i}\right) G_{0}(1, \iota, j, i)-(1-\lambda) m_{i} G_{0}(0, \iota, j, i) .
\end{aligned}
$$

Then, if we substitute from (10.16) into (10.15) and use the definition of $G_{k}\left(\iota^{c}, \iota^{p}, j, i\right)$, it follows that $\Delta(y, a)$ satisfies the matrix equation,

$$
\Delta(y, a)=b(y)+\beta C(a) \Delta(y, a) .
$$

By the definition of $h(\cdot ; y, a)$, it follows that $\Delta(y, a)$ is a fixed point of $h$. Therefore, (i) is true.

As regards (ii), it follows from (10.13) that $c_{i j}(a) / \pi_{i j} \in\left(1-\eta_{i j}\right)\left[1-N^{-1}, 1\right]$, where the lower endpoint is attained when money is always transferred and the upper endpoint when money is never transferred. Because $\eta_{i j}<1$, it follows that $c_{i j} / \pi_{i j} \in(0,1]$. That implies that $h(\cdot ; y, a)$ is increasing and satisfies discounting, Blackwell's sufficient conditions for contraction.

Claim (iii) is an obvious consequence of contraction. That is, if $h^{(n)}\left(\Delta(y, a) ; y^{\prime}, a^{\prime}\right)$ $\geq \Delta(y, a)$, then $h^{(n+1)}\left(\Delta(y, a) ; y^{\prime}, a^{\prime}\right)=h\left[h^{(n)}\left(\Delta(y, a) ; y^{\prime}, a^{\prime}\right) ; y^{\prime}, a^{\prime}\right] \geq h\left(\Delta(y, a) ; y^{\prime}, a^{\prime}\right)$ $\geq \Delta(y, a)$, where $h^{(n)}$ is the $n$-th iterate of $h$, where the first inequality follows from monotonicity of $h$ and the second inequality is the hypothesis, which 
serves as the initial condition for the induction step. But by the contraction property, $\lim _{n \rightarrow \infty} h^{(n)}\left(x ; y^{\prime}, a^{\prime}\right)=\Delta\left(y^{\prime}, a^{\prime}\right)$, which implies $\Delta\left(y^{\prime}, a^{\prime}\right) \geq \Delta(y, a)$.

From (10.14), $b(y)$ is increasing in $y$. That and claim (iii) imply claim (iv).

Lemma 2. If $(y, a)$ satisfies producer IR constraints, then $y\left(s, s^{\prime}, i\right) \leq \beta \max _{i} \Delta_{i}(y, a)$ $\leq y_{\max }^{1}$.

Proof. From equations (10.1) we have

$$
\begin{aligned}
N \delta_{i}(0, j)= & N \beta \Delta_{j}+\theta_{j i} z(y(0, j, i))+(1-\lambda)\left(1-m_{i}\right) z(y(0,0, i)) \\
& +(1-\lambda)\left[y(0,0, i)-a(0,0, i) \beta \Delta_{j}\right]+\theta_{j i}\left[y(0, j, i)-a(0, j, i) \beta \Delta_{j}\right] \\
& +\left(\lambda-\theta_{j i}\right)\left[y(j, 0, i)-a(j, 0, i) \beta \Delta_{j}\right] \\
N \delta_{i}(1, j)= & N \beta \Delta_{j}+\theta_{j i} z(y(j, j, i))+(1-\lambda)\left(1-m_{i}\right) z(y(j, 0, i)) \\
& +\lambda\left[y(j, j, i)-a(j, j, i) \beta \Delta_{j}\right]+(1-\lambda) m_{i}\left[y(0, j, i)-a(0, j, i) \beta \Delta_{j}\right] \\
& +(1-\lambda)\left(1-m_{i}\right)\left[y(j, 0, i)-a(j, 0, i) \beta \Delta_{j}\right]
\end{aligned}
$$

where $\Delta_{j}=\Delta_{j}(y, a)$. In each of these equations, the sum of the coefficients of $\beta \Delta_{j}$ is non-negative. Also, each coefficient of the function $z$ is non-negative. Therefore, if we let $K=\max _{i} \Delta_{i}(y, a)$ and let $\zeta=\max _{s, s^{\prime}, i} z\left(y\left(s, s^{\prime}, i\right)\right)$, we get the following inequalities:

$$
\begin{aligned}
N \delta_{i}(0, j) \leq & N \beta K+\left(1-m_{j}\right) \zeta+(1-\lambda)[y(0,0, i)-a(0,0, i) \beta K] \\
& +\theta_{j i}[y(0, j, i)-a(0, j, i) \beta K] \\
& +\left(\lambda-\theta_{j i}\right)[y(j, 0, i)-a(j, 0, i) \beta K] \\
N \delta_{i}(1, j) \leq & N \beta K+\left(1-m_{j}\right) \zeta+\lambda[y(j, j, i)-a(j, j, i) \beta K] \\
& +(1-\lambda) m_{i}[y(0, j, i)-a(0, j, i) \beta K] \\
& +(1-\lambda)\left(1-m_{i}\right)[y(j, 0, i)-a(j, 0, i) \beta K]
\end{aligned}
$$

where we used the definition of $\theta_{j i}$ to replace $\theta_{j i}+(1-\lambda)\left(1-m_{i}\right)$ by $1-m_{j}$. The producer IR constraints imply $y\left(s, s^{\prime}, i\right) \leq a\left(s, s^{\prime}, i\right) \beta K$, for all $s, s^{\prime}, i$. Therefore, $N \delta_{i}(\iota, j) \leq N \beta K+\left(1-m_{j}\right) \zeta$ for $\iota=0,1$. Now from (10.15), we have

$$
\Delta_{i} \leq \sum_{j=1}^{S} \pi_{i j}\left(1-\eta_{i j}\right)\left[\beta K+\frac{1}{N}\left(1-m_{j}\right) \zeta\right]
$$


Then because this holds for all $i$, and $\sum_{j} \pi_{i j}\left(1-\eta_{i j}\right) \leq 1$, and $1-m_{j} \leq 1-m_{1}$,

$$
K \leq \frac{\zeta\left(1-m_{1}\right)}{N(1-\beta)}
$$

By the definition of $y_{\max }^{1}$ and the assumption $y^{*} \geq y_{\max }^{1}$, we have

$$
\frac{\beta z\left(y^{*}\right)\left(1-m_{1}\right)}{N(1-\beta)} \leq y^{*} \text {. }
$$

Then since $\zeta \leq z\left(y^{*}\right)$ it follows that

$$
\beta K \leq \frac{\beta z\left(y^{*}\right)\left(1-m_{1}\right)}{N(1-\beta)} \leq y^{*} .
$$

Now because the function $z$ is increasing on the interval $\left[0, y^{*}\right]$ and because $y\left(s, s^{\prime}, i\right) \leq \beta K$, it follows that $\zeta \leq z(\beta K)$. Thus (10.21) implies

$$
\beta K \leq \frac{\beta z(\beta K)\left(1-m_{1}\right)}{N(1-\beta)} .
$$

This, in turn, implies $\beta K \leq y_{\max }^{1}$.

Proposition 1. If $(y, a)$ is optimal and satisfies $u\left(\beta \min _{s} \Delta_{s}(y, a)\right) \geq y_{\max }^{1}$, then (i) $S^{c}(i) \equiv \mathbb{S}_{+}$and $(y, a)$ satisfies (5.1)-(5.4); (ii) for each $i, S^{p}(i)$ is not empty; and (iii) if $\Delta_{j}(y, a)=\Delta_{k}(y, a)$, then for each $i$ either $j, k \in S^{p}(i)$ or $j, k \in \mathbb{S}_{+}-S^{p}(i)$.

Proof. Suppose that $(y, a)$ is optimal, but does not satisfy (i). Then consider $\left(y^{\prime}, a^{\prime}\right)$ given by (5.1)-(5.4), but with $\Delta(y, a)$ inserted on the right sides of (5.1)-(5.4) and with $S^{c}(i)$ replaced by $\mathbb{S}_{+}$.

Our first task is to show that $y^{\prime} \geq y$. For all but $Y^{c}(i)^{\prime}$, this follows immediately from the fact that each component of $y$ satisfies the relevant producer IR constraint. For $Y^{c}(i)^{\prime}$, we have,

$Y^{c}(i) \leq \frac{1}{\gamma_{i}^{1}\left(S^{c}(i)\right)} \sum_{j \in S^{c}(i)} \pi_{i j}\left(\lambda-\theta_{j i}\right) \beta \Delta_{j} \leq \frac{1}{\gamma_{i}^{1}\left(\mathbb{S}_{+}\right)} \sum_{j \in \mathbb{S}_{+}} \pi_{i j}\left(\lambda-\theta_{j i}\right) \beta \Delta_{j}=Y^{c}(i)^{\prime}$,

where the first inequality is by incentive feasibility of $y$ and where the second is lemma 7.

We next show that $\Delta\left(y^{\prime}, a^{\prime}\right) \geq \Delta(y, a)$. By the third part of lemma 8 , it is enough to show that $h_{i}\left(\Delta(y, a) ; y^{\prime}, a^{\prime}\right) \geq \Delta_{i}(y, a)=h_{i}(\Delta(y, a) ; y, a)$, where $h_{i}$ is the $i$-th component of the mapping defined in (10.12). Because $h_{i}$ is a sum of terms, we can deal one-at-a-time with the replacements given by (5.1)(5.4). Because $y^{\prime} \geq y$ and $h(x ; y, a)$ is increasing in $y$, we need only examine situations in which the replacement of $(y, a)$ by $\left(y^{\prime}, a^{\prime}\right)$ involves replacing a no-trade outcome by a trade outcome. 
(5.1)An informed consumer meets an uninformed producer:

Considering only the (5.1) substitution, (10.17) and the definition of $Y^{c}(i)^{\prime}$ imply that

$$
\begin{aligned}
\frac{N}{1-\lambda} \quad & {\left[h_{i}\left(\Delta(y, a) ; y^{\prime}, a^{\prime}\right)-h_{i}(\Delta(y, a) ; y, a)\right] } \\
= & \left(1-m_{i}\right) \sum_{j \in S^{c}(i)} \pi_{i j}\left(\lambda-\eta_{i j}\right)\left[u\left(Y^{c}(i)^{\prime}\right)-u\left(Y^{c}(i)\right)\right] \\
& +\left(1-m_{i}\right) \sum_{j \in \mathbb{S}_{+}-S^{c}(i)} \pi_{i j}\left(\lambda-\eta_{i j}\right)\left[u\left(Y^{c}(i)^{\prime}\right)-\beta \Delta_{j}\right] \\
& -\sum_{j \in S^{c}(i)} \pi_{i j}\left(\lambda-\theta_{i j}\right)\left[Y^{c}(i)-\beta \Delta_{j}\right] .
\end{aligned}
$$

Because $(y, a)$ satisfies the uninformed producer's IR constraint, $\sum_{j \in S^{c}(i)} \pi_{i j}(\lambda-$ $\left.\theta_{i j}\right)\left[Y^{c}(i)-\beta \Delta_{j}\right] \leq 0$. Therefore,

$$
\begin{gathered}
\frac{N}{(1-\lambda)\left(1-m_{i}\right)}\left[h_{i}\left(\Delta(y, a) ; y^{\prime}, a^{\prime}\right)-h_{i}(\Delta(y, a) ; y, a)\right] \geq \\
\sum_{j \in S^{c}(i)} \pi_{i j}\left(\lambda-\eta_{i j}\right)\left[u\left(Y^{c}(i)^{\prime}\right)-u\left(Y^{c}(i)\right)\right]+\sum_{j \in \mathbb{S}_{+}-S^{c}(i)} \pi_{i j}\left(\lambda-\eta_{i j}\right)\left[u\left(Y^{c}(i)^{\prime}\right)-\beta \Delta_{j}\right]
\end{gathered}
$$

The first term is nonnegative by $(10.25)$. The second term is nonnegative because $Y^{c}(i)^{\prime} \geq \beta \min _{s} \Delta_{s}$ and $u\left(\beta \min _{s} \Delta_{s}\right) \geq y_{\max }^{1} \geq \beta \max _{s} \Delta_{s}$ by hypothesis and lemma 2.

(5.2) An uninformed consumer meets an uninformed producer:

Here, by (10.17), the replacement of no trade by trade given by (5.2) implies

$$
\begin{gathered}
N\left[h_{i}\left(\Delta(y, a) ; y^{\prime}, a^{\prime}\right)-h_{i}(\Delta(y, a) ; y, a)\right]= \\
(1-\lambda)^{2}\left(1-m_{i}\right) \sum_{j=1}^{S} \pi_{i j}\left[u\left(y(0,0, i)^{\prime}\right)-\beta \Delta_{j}\right] \geq 0 .
\end{gathered}
$$

The equality follows from the definition of $y(0,0, i)^{\prime}$ and the inequality is implied by $y(0,0, i)^{\prime} \geq \beta \min _{s} \Delta_{s}$ and $u\left(\beta \min _{s} \Delta_{s}\right) \geq y_{\max }^{1} \geq \beta \max _{s} \Delta_{s}$.

(5.3) An informed consumer meets an informed producer

Here, by (10.17), the replacement of no trade by trade given by (5.3) implies $N\left[h_{i}\left(\Delta(y, a) ; y^{\prime}, a^{\prime}\right)-h_{i}(\Delta(y, a) ; y, a)\right]=\sum_{j} \pi_{i j}\left(\lambda-\eta_{i j}\right) \theta_{j i}\left[u\left(\beta \Delta_{j}\right)-\beta \Delta_{j}\right] \geq 0$,

where the summation is over some set of current states. 
We now have $y^{\prime} \geq y$ and $\Delta\left(y^{\prime}, a^{\prime}\right) \geq \Delta(y, a)$. It follows that $\left(y^{\prime}, a^{\prime}\right)$ satisfies all the producer IR constraints. Moreover, by lemma $2, \beta \Delta\left(y^{\prime}, a^{\prime}\right) \leq y_{\max }^{1}$. Therefore, $u\left(\beta \min _{s} \Delta_{s}\left(y^{\prime}, a^{\prime}\right)\right) \geq u\left(\beta \min _{s} \Delta_{s}(y, a)\right) \geq y_{\max }^{1} \geq \beta \max _{s} \Delta_{s}\left(y^{\prime}, a^{\prime}\right)$. This implies that $\left(y^{\prime}, a^{\prime}\right)$ satisfies all the consumer IR constraints. It also satisfies truth-telling for the consumer because it has trade in every state when the consumer is informed. But $\left(y^{\prime}, a^{\prime}\right)$ may or may not satisfy truth-telling for producers. If it does, then we have a contradiction because $y_{\max }^{1} \geq y^{\prime} \geq y$ and we have either increased output or replaced no-trade by trade, both of which increase $Z$.

If not, because $\Delta\left(y^{\prime}, a^{\prime}\right) \geq \Delta(y, a)$, then there is some previous state $i$ and some current state $r$ such that $a(0, r, i)=0$ and

$$
\beta \Delta_{r}\left(y^{\prime}, a^{\prime}\right)>Y^{p}(i)^{\prime} \geq \beta \Delta_{r}(y, a)
$$

where the second inequality holds because $(y, a)$ is incentive-feasible. That is, the informed producer would like to get money in additional states when responding to the incentives implied by $\Delta\left(y^{\prime}, a^{\prime}\right)$ as opposed to those implied by $\Delta(y, a)$. If so, then define a new mechanism $\left(y^{\prime \prime}, a^{\prime \prime}\right)$ that agrees with $\left(y^{\prime}, a^{\prime}\right)$ except that

$$
a(0, r, i)^{\prime \prime}=1 \text { and } y(0, r, i)^{\prime \prime}=Y^{p}(i)^{\prime} .
$$

for all pairs $r, i$ satisfying (10.29). This increases the number of states with trade when the producer has private information. By (10.17), the replacement of no-trade by trade in state $r$ implies

$$
\begin{gathered}
\frac{N}{1-\lambda}\left[h_{i}\left(\Delta(y, a) ; y^{\prime \prime}, a^{\prime \prime}\right)-h_{i}(\Delta(y, a) ; y, a)\right]= \\
\pi_{i r} \theta_{r i}\left[u\left(Y^{p}(i)^{\prime}\right)-\beta \Delta_{r}(y, a)\right]+\pi_{i r}\left(\lambda-\eta_{i r}\right) m_{i}\left[Y^{p}(i)^{\prime}-\beta \Delta_{r}(y, a)\right] \geq 0 .
\end{gathered}
$$

Therefore, by the third part of lemma $8, \Delta\left(y^{\prime \prime}, a^{\prime \prime}\right) \geq \Delta(y, a)$. Thus (by repeating the arguments given above for $\left.\left(y^{\prime}, a^{\prime}\right)\right)$, the mechanism $\left(y^{\prime \prime}, a^{\prime \prime}\right)$ satisfies all the constraints except possibly the producer's truth-telling constraints; if these are also satisfied then $\left(y^{\prime \prime}, a^{\prime \prime}\right)$ is incentive-feasible and has higher output and, therefore, higher welfare than $(y, a)$, contradicting the assumption that $(y, a)$ is optimal. Otherwise, we amend $\left(y^{\prime \prime}, a^{\prime \prime}\right)$ by adding states in which trade occurs. Since the producer's truth-telling constraints are satisfied if $S^{p}(i)=\mathbf{S}_{+}$, and since the number of states is finite, repetition of these steps must lead to a mechanism that satisfies the producer's truth-telling constraints, with higher output and therefore higher welfare than $(y, a)$, a contradiction. Thus, condition (i) is necessary for an optimum.

Now suppose $(y, a)$ is optimal, but $S^{p}(i)$ is empty. Let $k$ be such that $\Delta_{k}(y, a)=\max _{j} \Delta_{j}(y, a)$. Then consider $\left(y^{\prime}, a^{\prime}\right)$ that agrees with $(y, a)$ except that $a(0, k, i)^{\prime}=1$ and $y(0, k, i)^{\prime}=\beta \Delta_{k}(y, a)$. Then, as above, it can be shown that $\Delta\left(y^{\prime}, a^{\prime}\right) \geq \Delta(y, a)$, and it follows that $\left(y^{\prime}, a^{\prime}\right)$ gives higher welfare 
and satisfies all the constraints except perhaps the truth-telling constraint for producers. However, if it fails to satisfy that constraint, then there are two possibilities. One is that (10.29) might hold with respect to a previous state $i_{0} \neq i$ and, therefore, such that $S^{p}\left(i_{0}\right)^{\prime}=S^{p}\left(i_{0}\right)$. In that case we can apply the argument above which leads to a contradiction. The other is that

$$
\beta \Delta_{r}\left(y^{\prime}, a^{\prime}\right)>y(0, k, i)^{\prime}=\beta \Delta_{k}(y, a) \geq \beta \Delta_{r}(y, a)
$$

where the last inequality holds because $\Delta_{k}(y, a)=\max _{j} \Delta_{j}(y, a)$. But this, too, is a version of (10.29). Hence, again, we can define a new mechanism $\left(y^{\prime \prime}, a^{\prime \prime}\right)$ that agrees with $\left(y^{\prime}, a^{\prime}\right)$ except that $a(0, r, i)^{\prime \prime}=1$ and $y(0, r, i)^{\prime \prime}=\beta \Delta_{k}(y, a)$ for all states $r$ satisfying (10.32). Thus, in this case also, we can argue to a contradiction.

Finally, suppose $(y, a)$ is optimal but does not satisfy (iii). Then the optimum is such that $S^{p}(i)$ for some $i$ partitions states in such a way that there are two current states, say $j$ and $k$ with $\Delta_{j}=\Delta_{k}$ and $j \in S^{p}(i)$ and $k \notin S^{p}(i)$. By truth-telling, it follows that $\Delta_{j}=\min _{l \in S^{p}(i)} \Delta_{l}$. Therefore, by necessary condition (i), $(y, a)$ has $Y^{p}(i)=\beta \Delta_{j}$. Then amend $(y, a)$ by making the omitted state a trade state without changing output. The rest of the argument is exactly like the argument for necessary condition (i), and, therefore, produces a contradiction.

Lemma 3. Given $S^{p}(i)$ for each $i \in \mathbf{S}_{+}$, there exists a unique monetary mechanism that satisfies (5.1)-(5.4).

Proof. We will use the following result (see [4]): Suppose $f=\left(f^{1}, f^{2}, \ldots, f^{n}\right)$ is a function from $R^{n}$ to $R^{n}$ such that (i) $f$ is increasing; (ii) for each $i, f^{i}$ is a strictly concave function from $R^{n}$ to $R$; (iii) $f(0) \geq 0$; (iv) there is a positive vector $x^{a}$ such that $f\left(x^{a}\right)>x^{a} ;(\mathrm{v})$ there is a vector $x^{b}>x^{a}$ such that $f\left(x^{b}\right)<x^{b}$. Then there exists a unique positive vector $x$ such that $f(x)=x$. Moreover, $x \in\left(x^{a}, x^{b}\right)$.

The proof proceeds by defining a mapping that satisfies conditions (i)-(v) and each of whose fixed points is a monetary mechanism that satisfies (5.1)(5.4). Let $f$ from $R^{S}$ to $R^{S}$ be defined as follows. For $\Delta \in R^{S}$ let $y=g(\Delta)$ be the mapping defined by (5.1)-(5.4). Then define $f(\Delta) \equiv b(g(\Delta))+\beta C(a) \Delta$, where the vector $b$ and the matrix $C$ are as defined in (10.17). It follows that if $\Delta$ is a fixed point of $f$, then $g(\Delta)$ and the associated money transfers is a mechanism that satisfies (5.1)-(5.4) and vice versa. Therefore, for existence and uniqueness, the claims in the lemma, it is enough to show that $f(\Delta)$ satisfies conditions (i)-(v).

The mapping $g$ is increasing and $b$ is also increasing, so $f$ is increasing. Because the min function is concave, it follows that $g$ is concave. And because 
$b$ is concave in $y, b(g(\Delta))$, the composition of two increasing concave functions, is concave. Also, $f^{i}(\Delta)$ can be written in the form

$$
f^{i}(\Delta)=\frac{1}{N} \sum_{j=1}^{S} \pi_{i j}\left(\lambda-\eta_{i j}\right) \theta_{j i} u(y(j, j, i))+\Psi(\Delta),
$$

where $\Psi$ is a nonnegative concave function, $\pi_{i j}\left(\lambda-\eta_{i j}\right) \theta_{j i}$ is positive, and $y(j, j, i)=\frac{\Delta_{j}}{\beta}$. Because $u$ is strictly concave, this implies that $f^{i}$ is strictly concave.

Let $\Delta^{a}$ be the constant vector with $\Delta_{j}^{a}=\frac{\varepsilon}{\beta}, j \in \mathbf{S}_{+}$. Then

$$
f^{i}\left(\Delta^{a}\right) \geq \frac{u(\varepsilon)}{N} \sum_{j=1}^{S} \pi_{i j}\left(\lambda-\eta_{i j}\right) \theta_{j i} \geq \frac{u(\varepsilon)}{N} \pi_{i i} \lambda \theta_{i i}=\frac{u(\varepsilon)}{N} \pi_{i i} \lambda^{2}\left(1-m_{i}\right) .
$$

Because $u^{\prime}(0)=\infty$, the ratio $\frac{u(\varepsilon)}{\varepsilon}$ becomes arbitrarily large as $\varepsilon \rightarrow 0$. Choose $\varepsilon$ so that

Then

$$
\frac{u(\varepsilon)}{\varepsilon}>\frac{N}{\beta \pi_{i i} \lambda^{2}\left(1-m_{i}\right)}
$$

$$
f^{i}\left(\Delta^{a}\right)>\frac{\varepsilon}{\beta}=\Delta^{a}
$$

Thus $\Delta^{a}$ satisfies assumption (iv) of the fixed point theorem.

Let $\Delta^{b}$ be the constant vector with $\Delta_{j}^{b}=\frac{y^{*}}{\beta}, j \in \mathbf{S}_{+}$. (Recall that $u^{\prime}\left(y^{*}\right)=$ 1.) Then $f^{i}\left(\Delta^{b}\right)<\frac{y^{*}}{\beta}$ by essentially the argument in the proof of lemma 2 . Therefore, $\Delta^{b}$ satisfies assumption (v) of the fixed point theorem. Finally, because the mapping $f$ satisfies condition (iii) with equality, $f$ has a unique positive fixed point $\hat{\Delta}$.

Lemma 4. Let $S^{p}(i)$ for $i \in \mathbf{S}_{+}$be given. Let $\mathbf{P}=\left\{\Pi: \pi_{i i} \geq \alpha>0\right.$ for all $\left.i\right\}$. Then the unique monetary mechanism that satisfies (5.1)-(5.4) is continuous in $\Pi$ for $\Pi \in \mathbf{P}$.

Proof. Let $\Delta^{*}(\Pi)$ be the unique positive fixed point of $f(\Delta ; \Pi)$, where, for a given $\Pi \in \mathbf{P}, f$ is the mapping defined in the proof of lemma 3 . That is, $f(\Delta ; \Pi) \equiv b(g(\Delta))+\beta C(a) \Delta$, where $g$ is defined by (5.1)-(5.4). We now restrict the domain of $f$ to $\Delta \in\left[\Delta^{a}, \Delta^{b}\right]$, where $\Delta^{b}$ is the bound given in lemma 3 and where $\Delta^{a}$ is given by (10.35), but with $\pi_{i i}$ in (10.34) replaced by $\alpha$. So defined, the interval $\left[\Delta^{a}, \Delta^{b}\right]$ does not depend on $\Pi$. For a given $\Delta, f$ is continuous in $\Pi$, because it is a composite of two functions. each of which is continuous in the components of $\Pi$. And with $\Delta$ limited to the compact domain $\left[\Delta^{a}, \Delta^{b}\right]$, it follows that $f$ is uniformly continuous in $\Pi$. That is, for any $\varepsilon>0$, there 
exists a $\delta_{1}>0$ such that if $\left\|\Pi_{1}-\Pi_{2}\right\|<\delta_{1}$, then $\left\|f\left(\Delta ; \Pi_{1}\right)-f\left(\Delta ; \Pi_{2}\right)\right\|<\frac{\varepsilon}{3}$ for all $\Delta$.

Let $\Pi_{n}$ be a sequence converging to $\Pi$. Because the sequence $\Delta^{*}\left(\Pi_{n}\right)$ lies in a compact set, it has a convergent subsequence $\Delta^{*}\left(\Pi_{k}\right)$. Let $\hat{\Delta}$ be the limit of this subsequence. Then there exists a $\delta_{2}>0$ such that $\left\|\Pi_{k}-\Pi\right\|<\delta_{2}$ implies $\left\|f\left(\Delta^{*}\left(\Pi_{k}\right) ; \Pi_{k}\right)-\hat{\Delta}\right\|<\frac{\varepsilon}{3}$. Also, because $f$ is concave in $\Delta$, and, therefore, continuous in $\Delta$, there exists a $\delta_{3}>0$ such that $\left\|\Pi_{k}-\Pi\right\|<$ $\delta_{3}$ implies $\left\|f\left(\Delta^{*}\left(\Pi_{k}\right) ; \Pi\right)-f(\hat{\Delta} ; \Pi)\right\|<\frac{\varepsilon}{3}$. And by uniform continuity in $\Pi$, $\left\|\Pi_{k}-\Pi\right\|<\delta_{1}$ implies $\left\|f\left(\Delta^{*}\left(\Pi_{k}\right) ; \Pi_{k}\right)-f\left(\Delta^{*}\left(\Pi_{k}\right) ; \Pi\right)\right\|<\frac{\varepsilon}{3}$. Thus, if $k$ is such that $\left\|\Pi_{k}-\Pi\right\|<\min \left(\delta_{1}, \delta_{2}, \delta_{3}\right)$, then

$$
\begin{gathered}
\|f(\hat{\Delta} ; \Pi)-\hat{\Delta}\| \leq\left\|f(\hat{\Delta} ; \Pi)-f\left(\Delta^{*}\left(\Pi_{k}\right) ; \Pi\right)\right\|+ \\
\left\|f\left(\Delta^{*}\left(\Pi_{k}\right) ; \Pi\right)-f\left(\Delta^{*}\left(\Pi_{k}\right) ; \Pi_{k}\right)\right\|+\left\|f\left(\Delta^{*}\left(\Pi_{k}\right) ; \Pi_{k}\right)-\hat{\Delta}\right\|<\varepsilon .
\end{gathered}
$$

Because this holds for any $\varepsilon>0, f(\hat{\Delta} ; \Pi)=\hat{\Delta}$. And because $f(\hat{\Delta} ; \Pi)$ has a unique positive fixed point, it follows that $\hat{\Delta}=\Delta^{*}(\Pi)$. Finally, if that original sequence $\Delta^{*}\left(\Pi_{n}\right)$ does not converge to $\Delta^{*}(\Pi)$, then it must have a subsequence that is bounded away from $\Delta^{*}(\Pi)$. But that subsequence must in turn have a convergent subsequence. By the argument just given, the limit of that subsequence can only be $\Delta^{*}(\Pi)$. Thus, $\Delta^{*}(\Pi)$ is continuous.

Proposition 2. If the optimum satisfies $u\left(\beta \min _{s} \Delta_{s}\right) \geq y_{\max }^{1}$, then the maximized objective is continuous in $\Pi$ for $\Pi$ such that $\pi_{i j}$ is positive and $\pi_{i i}$ is bounded away from 0 .

Proof. For a given transition matrix $\Pi$, let $\Omega(\Pi)$ be the set of mechanisms identified in lemma 3. Also, let $\Omega^{*}(\Pi)$ be the subset of $\Omega(\Pi)$ that is incentivefeasible. Because the always-trade mechanism $\left(S^{p}(i) \equiv \mathbf{S}_{+}\right)$is incentivefeasible and is in $\Omega(\Pi), \Omega^{*}(\Pi)$ is not empty.

Let $\Pi_{n} \rightarrow \Pi^{0}$, and let $Z_{n}^{*}$ be the optimal welfare for $\Pi=\Pi_{n}$, with $Z_{n}^{*}=$ $\zeta\left(y\left(\Pi_{n}\right), a\left(\Pi_{n}\right)\right)$, where the function $\zeta$ is defined by equation (4.13); also let $Z_{0}^{*}$ be the optimal welfare for $\Pi=\Pi_{0}$, with $Z_{0}^{*}=\zeta\left(y\left(\Pi^{0}\right), a\left(\Pi^{0}\right)\right)$.

Suppose $\limsup _{n \rightarrow \infty} Z_{n}^{*}>Z_{0}^{*}$. Then there is a positive number $\varepsilon$ and a subsequence $Z_{n_{j}}^{*}$ such that $Z_{n_{j}}^{*}>Z_{0}^{*}+\varepsilon$ for all $j$, with $Z_{n_{j}}^{*}=\zeta\left(y\left(\Pi_{n_{j}}\right), a\left(\Pi_{n_{j}}\right)\right)$. Because the set of possible $a^{\prime} s$ is finite (there is a finite number of possible specifications of the set $S^{p}(i)$ for each $\left.i \in \mathbf{S}_{+}\right)$, there must be at least one $a$ that occurs infinitely often in this subsequence. Denote such an $a$ by $\hat{a}$. Then we have, $\zeta\left(\hat{y}\left(\Pi_{n_{j}}\right), \hat{a}\right)>Z_{0}^{*}+\varepsilon$ for all $j$, where $\hat{y}\left(\Pi_{n_{j}}\right)$ denotes the unique lemma 3 mechanism for $a=\hat{a}$. Then, by lemma $4, \zeta\left(\hat{y}\left(\Pi^{0}\right), \hat{a}\right)>Z_{0}^{*}$. Therefore, the optimality of $Z_{0}^{*}$ implies $\left(\hat{y}\left(\Pi^{0}\right), \hat{a}\right) \notin \Omega^{*}\left(\Pi^{0}\right)$. But since $\left(\hat{y}\left(\Pi_{n_{j}}\right), \hat{a}\right) \in \Omega^{*}\left(\Pi_{n_{j}}\right)$, we have $\Delta_{r}\left(\hat{y}\left(\Pi_{n_{j}}\right), \hat{a}\right) \leq \Delta_{s}\left(\hat{y}\left(\Pi_{n_{j}}\right), \hat{a}\right)$ for all $i, r, s$ such that $r \notin \hat{S}^{p}(i)$ and 
$s \in \hat{S}^{p}(i)$, where $\hat{S}^{p}(i)$ is the set $S^{p}(i)$ that corresponds to $\hat{a}$. Because, again by lemma 4 , these inequalities are preserved in the limit as $\Pi_{n_{j}} \rightarrow \Pi^{0}$, we have $\left(\hat{y}\left(\Pi^{0}\right), \hat{a}\right) \in \Omega^{*}\left(\Pi^{0}\right)$, a contradiction. Therefore, $\lim _{\sup _{n \rightarrow \infty}} Z_{n}^{*} \leq Z_{0}^{*}$.

Let $a=\tilde{a}$ be optimal at $\Pi=\Pi^{0}$. By part (iii) of Proposition 1 we know that, for all $i \in \mathbf{S}_{+}, r \notin \tilde{S}^{p}(i)$ and $s \in \tilde{S}^{p}(i)$ implies $\Delta_{r}\left(\tilde{y}\left(\Pi^{0}\right), \tilde{a}\right)<\Delta_{s}\left(\tilde{y}\left(\Pi^{0}\right), \tilde{a}\right)$, where $\tilde{y}(\Pi)$ denotes the unique lemma 3 mechanism for $a=\tilde{a}$. Lemma 4 then implies that $\left(\tilde{y}\left(\Pi_{n}\right), \tilde{a}\right) \in \Omega^{*}\left(\Pi_{n}\right)$ for $n$ sufficiently large. Thus $Z_{n}^{*} \geq$ $\zeta\left(\tilde{y}\left(\Pi_{n}\right), \tilde{a}\right)$, which implies $\liminf _{n \rightarrow \infty} Z_{n}^{*} \geq Z_{0}^{*}$.

It follows that $\lim _{n \rightarrow \infty} Z_{n}^{*}=Z_{0}^{*}$.

Proposition 3. Let $\left\{\Pi_{k}\right\} \rightarrow I$ (the identity matrix) and be such that the corresponding sequence of invariant probability vectors $\left\{p_{k}\right\}$ converges to a strictly positive vector. Assume that the optimum for any $\Pi$ in the neighborhood of $\Pi=I$ satisfies $u\left(\beta \min _{s} \Delta_{s}\right) \geq y_{\max }^{1}$. There exists $K$ such that if $k>K$, then the optimum for $\Pi_{k}$ has no trade between informed producers and uninformed consumers whenever the current state, $j$, exceeds the previous state, $i$.

Proof. Let $\left\{\Pi_{k}\right\} \rightarrow I$ be given and let $\left\{p_{k}\right\} \rightarrow p^{\prime}$. For $\Pi=I$, define the function, $Z(I)$, to be $Z$ with $p=p^{\prime}$. The maximum of $Z(I)$ over incentivefeasible $(y, a)$ is $y\left(\iota^{c} i, \iota^{p} i, i\right)=y_{\max }^{i}$. (When $\Pi=I$, the maximum is obtained state by state and for each state, the result is as described in Section 3.) Denote that maximum $Z^{*}(I)$.

Now let $S^{p}(i)^{\prime}=\{1,2, \ldots, i\}$ for each $i$, and let $y^{k}$ and $\Delta^{k}$ denote the unique monetary mechanism that satisfies (5.1)-(5.4) of Proposition 1 when $S^{p}(i)=$ $S^{p}(i)^{\prime}$ and $\Pi=\Pi_{k}$; also, let $y^{\infty}$ and $\Delta^{\infty}$ denote $y^{k}$ and $\Delta^{k}$ for $\Pi=I$. Then, $y^{\infty}\left(\iota^{c} i, \iota^{p} i, i\right)=y_{\max }^{i}$ and $\Delta_{i}^{\infty}=y_{\max }^{i} / \beta$. (This is verified by inserting these expressions into (5.1)-(5.4) and (10.17) and noticing that they hold.) Then, by lemma $4, \lim _{k \rightarrow \infty} y^{k}\left(\iota^{c} i, \iota^{p} i, i\right)=y_{\max }^{i}$ and $\lim _{k \rightarrow \infty} \Delta_{i}^{k}=y_{\max }^{i} / \beta$. That, in turn, implies that if $k$ is sufficiently large, then $y_{k}^{\prime}$ is incentive feasible. (This follows because the limit result implies that if $k$ is large, then $\Delta_{i}^{k}>\Delta_{i+1}^{k}$.) Now let $Z^{*}(\Pi)$ denote the maximized objective. It follows that for any $\varepsilon>0$, there exists $K$ such that $k>K$ implies $Z^{*}\left(\Pi_{k}\right)>Z^{*}(I)-\varepsilon$. We now show that if $\Pi_{k}$ is close enough to $I$, then a mechanism which has trade when the current state $j$ and previous state $i$ satisfy $j>i$ gives a value of the objective that is less than $Z^{*}(I)-\varepsilon$, and, therefore, cannot be optimal.

If $(y, a)$ satisfies producer IR constraints, then

$$
\lim _{\Pi \rightarrow I} \Delta_{i}(y, a ; \Pi) \leq y_{\max }^{i} / \beta .
$$


This follows by taking the limit of $\Delta_{i}(y, a)$ as given by (10.17) and noting two things: only terms of the form $\lim y\left(\iota^{c} i, \iota^{p} i, i\right)$ and $\lim \left[a\left(\iota^{c} i, \iota^{p} i, i\right) \beta \Delta_{i}\right]$ appear and the IR constraints imply $\lim y\left(\iota^{c} i, \iota^{p} i, i\right) \leq \lim \left[a\left(\iota^{c} i, \iota^{p} i, i\right) \beta \Delta_{i}\right]$. Now suppose by contradiction that the no-trade claim is false. Then there exists a subsequence of the given sequence $\left\{\Pi_{k}\right\}$, say $\left\{\Pi_{n}\right\}$, such that $\left\{\Pi_{n}\right\} \rightarrow I$ and for each $n$ the optimum has trade in some current state $j$, where $j>i$, the previous state. Let $Z^{\prime \prime}\left(\Pi_{n}\right)$ denote the implied value of $Z$. The producer truth-telling constraint and (10.37) imply that for sufficiently large $n$, either $a(0, i, i)^{n}=0$ or $y(0, i, i)^{n} \leq y_{\max }^{j}+\varepsilon_{0}$, where $j>i$ and where $\varepsilon_{0}>0$ but is otherwise arbitrary. (Recall that $y_{\max }^{j}<y_{\max }^{i}$ if $j>i$ and that $y_{\max }^{i}$ depends on $m_{i}$, but not on $\Pi$.) But (10.37) also implies that for sufficiently large $n, y\left(\iota^{c} i, \iota^{p} i, i\right)^{n} \leq y_{\max }^{i}+\varepsilon_{i}$, where, again, $\varepsilon_{i}>0$ but is otherwise arbitrary. Because a component of $y$ appears with a non-vanishing coefficient in $\lim _{n \rightarrow \infty} Z\left(\Pi_{n}\right)$ if and only if it has the form $y\left(\iota^{c} i, \iota^{p} i, i\right)$, it follows that $\lim \sup \left\{Z^{\prime \prime}\left(\Pi_{k}\right)\right\}<Z^{*}(I)$, a contradiction.

Lemma 5. Let $Z\left(y^{k}\right)$ be ex ante welfare implied by $y^{k}$ as given by (5.5). Let $Z_{\text {min }}$ be the ex ante welfare implied by some incentive-feasible mechanism. If there exists $Z_{\min }$ such that $Z\left(y^{k}\right) \leq Z_{\text {min }}$ for each $k \in \mathbf{S}_{+}$, then any optimum satisfies $u\left(\beta \min _{s} \Delta_{s}\right) \geq y_{\max }^{1}$.

Proof. If the optimum violates $u\left(\beta \min _{s} \Delta_{s}\right) \geq y_{\max }^{1}$, then for some $k \in \mathbf{S}_{+}$, $\beta \Delta_{k}<u^{-1}\left(y_{\max }^{1}\right)$. (Notice that $u^{-1}\left(y_{\max }^{1}\right)<y_{\max }^{1}$.) If so, then $y^{k}$ is strictly greater than any optimal $y$. For $y^{k}(j, 0, i)$, this follows from lemma 2. For $y^{k}(j, j, i)$ and $y^{k}(0,0, i)$, it follows directly from the respective producer individual rationality constraints. Finally, for $y^{k}(0, j, i)$, consider the second inequality in (4.8), which is the individual rationality constraint for informed producers. If $j \in S^{p}(i)$, then the minimum is no greater than $\beta \Delta_{j}^{k}$. If $j \notin S^{p}(i)$, then $y(0, j, i)=0$. In either case, we get $y(0, j, i) \leq y^{k}(0, j, i)$. Therefore, $Z\left(y^{k}\right)$ is strictly greater than the optimum $Z$. But if $Z\left(y^{k}\right) \leq Z_{\text {min }}$ for each $k \in \mathbf{S}_{+}$, then we have a contradiction and the optimum cannot satisfy $u\left(\beta \min _{s} \Delta_{s}\right)<y_{\max }^{1}$.

Lemma 6. There exists $\varepsilon>0$ such that if $m_{S}-m_{1}<\varepsilon$, then the hypothesis of lemma 5 holds.

Proof. Let $\left(y_{x}, 1\right)$ denote an always-trade mechanism with $y_{x}\left(s, s^{\prime}, i\right) \equiv x$, a mechanism in which trade occurs in every trade meeting and output is the same in every trade meeting. For such a mechanism,

$$
c_{i j}(a)=\left(1-\frac{1}{N}\right) \pi_{i j}\left(1-\eta_{i j}\right)
$$


and

$$
\begin{gathered}
N b_{i}\left(y_{x}\right)=(1-\lambda) \sum_{j} \pi_{i j}\left[\left(1-m_{j}\right) u(x)+m_{j} x\right]+ \\
\sum_{j} \pi_{i j}\left(\lambda-\eta_{i j}\right)\left[\left(1-m_{j}\right) u(x)+m_{j} x\right]= \\
\sum_{j} \pi_{i j}\left(1-\eta_{i j}\right)\left[\left(1-m_{j}\right) u(x)+m_{j} x\right]
\end{gathered}
$$

where the first equality in (10.39) comes from using the definition of $\theta_{j i}$ to combine, in turn, the first two lines and the last two lines of the expression for $N b_{i}(y)$ (see (10.14)). If $u(x) \geq x$, which is implied by $x \leq y_{\max }^{1}$, then

$$
N b_{i}\left(y_{x}\right) \geq\left[\left(1-m_{S}\right) u(x)+m_{S} x\right] \Phi(i)
$$

where $\Phi(i) \equiv \sum_{j} \pi_{i j}\left(1-\eta_{i j}\right)$. Now let $L=\min _{i} \Delta_{i}\left(y_{x}, 1\right)$. Then by $(10.17), L$ satisfies

$$
N L \geq N \min _{i} b_{i}\left(y_{x}\right)+(N-1) \beta L \min _{i} \Phi(i) .
$$

Therefore, by (10.40), $L$ satisfies

$$
N L \geq\left[\left(1-m_{S}\right) u(x)+m_{S} x\right] \Phi+(N-1) \beta L \Phi
$$

where $\Phi \equiv \min _{i} \Phi(i)$. Suppose, then, that we take for $x$ the positive solution to $x=\beta L$ and (10.42) at equality - namely, the unique positive solution for $x$ to

$$
x=\frac{\beta \Phi z(x)\left(1-m_{S}\right)}{(1-\beta \Phi) N} .
$$

(Notice that this solution is less than $y_{\max }^{S}$ because $\Phi<1$. In fact, it is $y_{\max }^{S}$ for an economy with a discount factor $\beta^{\prime}=\beta \Phi<\beta$.) By construction, then, $\left(y_{x}, 1\right)$ with $x$ given by the positive solution to (10.43) satisfies producer individual-rationality constraints. We next have to satisfy consumer individual-rationality constraints.

Let $K=\max _{i} \Delta_{i}\left(y_{x}, 1\right)$. Then by (10.17), $K$ satisfies

$$
N K \leq N \max _{i} b_{i}\left(y_{x}\right)+(N-1) \beta K \max _{i} \Phi(i) .
$$

Therefore, by the same reasoning that led to (10.40), $K$ satisfies

$$
N K \leq\left[\left(1-m_{1}\right) u(x)+m_{1} x\right]+(N-1) \beta K,
$$

where we have taken unity to be an upper bound on $\Phi(i)$. With $x$ given by the positive solution to $(10.43)$, it follows that $K \leq y_{\max }^{1} / \beta$. Therefore, it is sufficient for satisfaction of consumer individual-rationality constraints for the mechanism $\left(y_{x}, 1\right)$ with $x$ given by the positive solution to (10.43) that $u(x) \geq y_{\max }^{1}$. 
Because truth-telling is vacuous for the mechanism $\left(y_{x}, 1\right)$, that mechanism is incentive-feasible provided $u(x) \geq y_{\max }^{1}$, which is a condition that is easy to check. If it holds, then $Z\left(y_{x}\right)$ is a possible magnitude for $Z_{\min }$.

To complete the proof, we first show that $x \rightarrow y_{\max }^{1}$ as $m_{S}-m_{1} \rightarrow 0$. From (10.3) it follows that $\eta_{i j} \rightarrow 0$ as $m_{S}-m_{1} \rightarrow 0$. Therefore, $\Phi(i) \rightarrow 1$ as $m_{S}-m_{1} \rightarrow 0$. It follows that as $m_{S}-m_{1} \rightarrow 0, \Phi \rightarrow 1$ and, hence, by the definition of $x$ that $x \rightarrow y_{\max }^{1}$. That is, as $\Phi \rightarrow 1$ and $m_{S} \rightarrow m_{1}$ in (10.43), then the unique positive solution to (10.43) approaches the unique positive solution to (3.1) for $m_{i}=m_{1}$. Now, for $x$ sufficiently close to $y_{\max }^{1}$, we have $u(x) \geq y_{\max }^{1}$. Finally, for $x$ sufficiently close to $y_{\max }^{1}$, it follows that the hypothesis of lemma 5 holds.

\section{REFERENCES}

[1] R. J. Barro, ed., Modern Business Cycle Theory. Harvard University Press, 1989.

[2] A. Berentsen, M. Molico, and R. Wright, Indivisibilities, lotteries, and monetary exchange, Journal of Economic Theory, 2001 (pp. 1-25, doi:10.1006/jeth.2000.2689).

[3] L. Jones and R. Manuelli, Volatile policy and private information: the case of monetary shocks. Journal of Economic Theory, forthcoming.

[4] J. Kennan, Uniqueness of Positive Fixed Points for Increasing Concave Functions on $\mathbb{R}^{n}$ : An Elementary Result, http://www.ssc.wisc.edu/ jkennan/research/FixedPoint.htm, 2000.

[5] N. Kocherlakota, Money is memory. Journal of Economic Theory, 81 (1998) 232-251.

[6] R.E. Lucas Jr., Expectations and the neutrality of money. Journal of Economic Theory, 4 (1972) 103-124.

[7] R.E. Lucas Jr., Nobel Lecture: Monetary Neutrality. Journal of Political Economy, 104 (August 1996) 661-682.

[8] S. Shi, Money and prices: a model of search and bargaining. Journal of Economic Theory, 67 (1995) 467-498.

[9] A. Trejos and R. Wright, Search, bargaining, money and prices. Journal of Political Economy, 103 (1995) 118-141.

[10] N. Wallace, Lucas's signal-extraction model: a finite state exposition with aggregate real shocks. Journal of Monetary Economics, 30 (1992) 433-447.

[11] N. Wallace, Short-run and long-run effects of changes in money in a random-matching model. Journal of Political Economy, 105 (1997), 1293-1307.

[12] N. Wallace, Introduction to modeling money and studying monetary policy, Journal of Economic Theory, 81 (August 1998), 223-231.

Current address: Department of Economics, University of Wisconsin, 1180 Observatory Drive, Madison, WI 53706

E-mail address: jkennan@ssc.wisc.edu

URL: http://www.ssc.wisc.edu/ jkennan 